\title{
Anuran amphibians in state of Paraná, southern Brazil
}

\author{
Manuela Santos-Pereira ${ }^{I}{ }^{*}$, José P. Pombal Jr. ${ }^{2}$ \& Carlos Frederico D. Rocha ${ }^{I}$ \\ ${ }^{1}$ Universidade do Estado do Rio de Janeiro, Ecologia, Rua São Francisco Xavier, 524, \\ Rio de Janeiro, RJ, Brasil \\ ${ }^{2}$ Universidade Federal do Rio de Janeiro, Museu Nacional, Departamento de Vertebrados, \\ Rio de Janeiro, RJ, Brasil \\ *Corresponding author: Manuela Santos-Pereira, e-mail: herpeto.pereira@gmail.com
}

SANTOS-PEREIRA, M., POMBAL Jr., J.P., ROCHA, C.F.D. Anuran amphibians in state of Paraná, southern Brazil. Biota Neotropica. 18(3): e20170322. http://dx.doi.org/10.1590/1676-0611-BN-2017-0322

\begin{abstract}
The state of Paraná, located in southern Brazil, was originally covered almost entirely by the Atlantic Forest biome, with some areas of Cerrado savanna. In the present day, little of this natural vegetation remains, mostly remnants of Atlantic Forest located in the coastal zone. While some data are available on the anurans of the state of Paraná, no complete list has yet been published, which may hamper the understanding of its potential anuran diversity and limit the development of adequate conservation measures. To rectify this situation, we elaborated a list of the anuran species that occur in state of Paraná, based on records obtained from published sources. We recorded a total of 137 anuran species, distributed in 13 families. Nineteen of these species are endemic to the state of Paraná and five are included in the red lists of the state of Paraná, Brazil and/or the IUCN. Two anuran species were categorized as Near Threatened by the IUCN and 27 species were listed as Data Deficient in one or more lists. According to IUCN $49.6 \%$ of the anuran species recorded had their population trends stable, $19 \%$ in declined, only $1.4 \%$ is increased and $20.4 \%$ had your population trends unknown. We also recorded the occurrence in Paraná of the exotic invader anuran Lithobates catesbeianus. We consider our list of species to be a relatively reliable estimate of the anuran diversity of the Brazilian state of Paraná, although new species records are expected, mainly because there are many regions that have not yet been sampled.
\end{abstract}

Keywords: Atlantic Forest, checklist, Cerrado, inventory, species richness.

\section{Anfíbios anuros do estado do Paraná, sul do Brasil}

Resumo: O estado do Paraná, localizado no sul do Brasil, originalmente possuía praticamente toda sua área coberta pelo bioma Mata Atlântica com algumas porções do bioma Cerrado. Atualmente, pouco resta das formações destes biomas, sendo a maior parte remanescentes florestais de Mata Atlântica, localizados na região costeira do Estado. Apesar de haver estudos sobre anuros no estado do Paraná, até então nenhum deles proveu uma lista dos anuros com ocorrência no Estado, o que pode dificultar o conhecimento sobre a potencial diversidade de anuros e restringir medidas de conservação. A partir dessa lacuna, elaboramos uma lista das espécies de anuros com registro para o Paraná, a partir de dados de estudos publicados. Registramos um total de 137 espécies de anuros, pertencentes a 13 famílias. Dentre estas, 19 espécies de anuros são endêmicas do estado do Paraná e cinco encontram-se relacionadas sob algum grau de ameaça de extinção na lista vermelha do estado do Paraná, do Brasil e/ou da lista global da IUCN. Duas espécies de anuros foram categorizadas como Quase Ameaçada pela IUCN e 27 espécies foram listadas como Dados Insuficientes nestas listas. De acordo com a IUCN, 49,6\% das espécies de anuros registradas apresentaram tendências populacionais estáveis, 19\% diminuíram, apenas 1,4\% aumentaram e 20,4\% apresentaram tendências de sua populações desconhecidas. Registramos também a ocorrência do anuro exótico e invasor Lithobates catesbeianus para diferentes áreas do estado do Paraná. Consideramos nossa lista de espécies uma estimativa relativamente confiável da diversidade de anuros do estado brasileiro do Paraná, embora sejam esperados novos registros de espécies, principalmente porque existem muitas regiões que ainda não foram amostradas.

Palavras-chave: Mata Atlântica, lista de espécies, Cerrado, inventário, riqueza de espécies. 


\section{Introduction}

Currently 6836 species of anuran amphibians are known to exist worldwide (Frost 2017). Just under a sixth (15.2\%; 1039 species) of this total is found in Brazil (Segalla et al. 2016). This high species richness is partly related to the continental dimensions of the country and its considerable variation in altitude, as well as the enormous variety of tropical and subtropical habitats and ecosystems found in the different Brazilian biomes (Araújo et al. 2009), which provide a diversity of environments appropriate for anuran populations. In the Brazilian state of Paraná, most of these ecosystems are associated with the Atlantic Forest biome, although some areas of the Cerrado biome are also found in the state. Both these biomes have been classified as world biodiversity hotspots (Myers et al. 2000). Based on the modeling of the distribution data available for anuran species, Toledo \& Batista (2012) estimated that as many as 147 anuran species may potentially occur in the state. Crivellari et al. (2014) recently listed the anurans that occur in the southern grasslands of Paraná, citing a total of 61 species. Up until now, however, no complete list of the anurans known to occur in the state of Paraná has been published.

While species lists for the country as a whole or for its different political divisions are important for the understanding of their biodiversity and provide an additional tool for the development of conservation measures, knowledge on the diversity of most groups of animals, through studies that provide species lists, is still incipient for most Brazilian states. Currently, lists of anuran amphibians are only available for the Brazilian states of Alagoas (Almeida et al. 2016), Espírito Santo (Almeida et al. 2011), Piauí (Roberto et al. 2013), Rio de Janeiro (Rocha et al. 2004), Rio Grande do Sul (Machado \& Maltchik 2007), and São Paulo (Rossa-Feres et al. 2011), less than a quarter $(23.1 \%)$ of the total number of Brazilian states.

In the present study, we estimated the number of anuran taxa that occur in the Brazilian state of Paraná based on a compilation of published records. We identified the endemic species, the taxa listed as threatened, near threatened and data deficient and the population trend of each species (sensu IUCN 2017). We also mapped the localities in Paraná where anuran inventories have been conducted.

\section{Material and Methods}

To identify the anuran species that occur in the Brazilian state of Paraná, we considered data obtained from papers published in journals based on a search of the Web of Science, Scielo, Scopus, and Google Scholar databases. The search terms used to identify the papers were amphib* AND Paraná, anur* AND Paraná and frog* AND Paraná. We consulted the papers cited as references in these articles. We terminated the search in November 06, 2017. We also did a supplementary search on the website SpeciesLink (2017) to know if there was still some anuran species collected in the state of Paraná that had not been registered by us through the publications accessed. To identify the endemic species of the Paraná state and the Atlantic Forest, we analyzed the known geographic distribution of each recorded species by Frost (2017). The current conservation status of each species was obtained from the Red List of Threatened Fauna of the state of Paraná (Segalla \& Langone 2004), the Red List of Threatened Brazilian Fauna (MMA 2014), and the online version of the IUCN Red List of Threatened Species (IUCN
2017). The IUCN website was accessed to know the population trends of the anurans recorded in the present study (IUCN 2017). We followed the taxonomic nomenclature of Frost (2017).

\section{Results}

Based on the combined database (Table 1), we recorded 137 anuran species for the state of Paraná, belonging to 13 families: Hylidae $(\mathrm{n}=$ 58 species), Leptodactylidae (26), Brachycephalidae (15), Bufonidae (12), Cycloramphidae (7), Hylodidae (5), Odontophrynidae (5), Mycrohylidae (3), Hemiphractidae (2), Alsodidae (1), Centrolenidae (1), Craugastoridae (1), and Ranidae (1).

The Hylidae family was the richest in species (58 species, $42.3 \%$ of the total), while Alsodidae, Centrolenidae, Craugastoridae, Hemiphractidae and Ranidae were the least diverse, each one represented by a single species $(0.7 \%$ of the total) (Table 1$)$. The most diverse genus was Boana, with 13 species, $9.5 \%$ of the total recorded for the state. The most frequently recorded species in published studies were Boana faber (Wied-Neuwied, 1821) and Scinax fuscovarius (Lutz, 1925), each one recorded in 18 studies, and Dendropsophus minutus (Peters, 1872) and Ischnocnema henselii (Peters, 1870), both recorded in 17 studies. By contrast, out of 137 recorded species, 32 (23.3\%) were cited in only a single study, nine of them (28.1\%) are endemic to Paraná, and a further eight (25\%) are also found only in the neighboring states of Santa Catarina or São Paulo.

Out of 137 species recorded in this study (Table 1), 19 (13.9\%) are endemic to the state of Paraná: Brachycephalidae: Brachycephalus brunneus Ribeiro, Alves, Haddad \& Reis, 2005 (Pico Caratuva, Campina Grande do Sul municipality), B. coloratus Ribeiro, Blackburn, Stanley, Pie \& Bornschein, 2017 (Serra da Baitaca, Piraquara municipality), $B$. curupira Ribeiro, Blackburn, Stanley, Pie \& Bornschein, 2017 (Serra do Salto, São José dos Pinhais municipality), B. ferruginus Alves, Ribeiro, Haddad \& Reis, 2006 (Pico do Marumbi, Morretes municipality), B. izecksohni Ribeiro, Alves, Haddad \& Reis, 2005 (Pico Caratuva, Campina Grande do Sul municipality), B. leopardus Ribeiro, Firkowski \& Pie, 2015 (Serra do Araçatuda, Tijucas do Sul municipality; Morro dos Perdidos, Guaratuba municipality), B. pernix Pombal, Wistuba \& Bornschein, 1998 (Quatro Barras, Morretes and São José dos Pinhais municipality), B. pombali Alves, Ribeiro, Haddad \& Reis, 2006 (Pico da Igreja, Guaratuba municipality), B. tridactylus Garey, Lima, Hartmann \& Haddad, 2012 (Pico do Morato, Guaraqueçaba municipality), Ischnocnema paranaensis (Langone \& Segalla, 1996) (next to Pico do Paraná, Antonina municipality) and I. sambaqui (Castanho \& Haddad, 2000) (Guaraqueçaba and Morretes municipality); Bufonidae: Dendrophryniscus stawiarskyi Izecksohn, 1994 (Bituruna municipality), Melanophryniscus alipioi Langone, Segalla, Bornschein \& de Sá, 2008 (Campina Grande do Sul municipality) and M. vilavelhensis Steinbach-Padilha, 2008 (Ponta Grossa municipality); Cycloramphidae: Cycloramphus duseni (Anderson, 1914) (Ipiranga municipality) and C. mirandaribeiroi Heyer, 1983 (São João da Graciosa, Morretes municipality); Hylidae: Bokermannohyla langei (Bokermann, 1965) (Morretes municipality) and Boana jaguariaivensis (Caramaschi, Cruz \& Segalla, 2010) (Jaguariaíva municipality); Leptodactylidae: Physalaemus insperatus Cruz, Cassini \& Caramaschi, 2008 (Guaratuba municipality). 
Table 1. Anuran amphibians in the state of Paraná, southern Brazil, including geographical distribution in Brazil, endemism for the state of Paraná (both sensu Frost 2017), and conservation status [according to Red List of Threatened Fauna of the state of Paraná (Segalla \& Langone 2004), Red List of Threatened Brazilian Fauna (MMA 2014), and the IUCN Red List of Threatened Species (2017)]. Population trend of anurans follows IUCN (2017): $\mathrm{S}=\mathrm{stable}, \mathrm{D}=\mathrm{decreasing}, \mathrm{I}=$ increasing and U = unknown. Source of records: 1, Affonso \& Delariva (2012); 2-3, Affonso et al. (2011, 2014); 4, Alves et al. (2006); 5, Antonucci et al. (2011); 6, Armstrong \& Conte (2010); 7, Baldo et al. (2008); 8, Benarde \& Anjos (1999); 9, Bernarde \& Machado (2001); 10, Bokermann (1965); 11, Bornschein et al. (2015a); 12, Bornschein et al. (2015b); 13, Bornschein et al. (2016b); 14, Caramaschi \& Cruz (2002); 15, Caramaschi \& Rodrigues (2007); 16, Caramaschi et al. (2010); 17, Castanho \& Haddad (2000); 18-19, Clemente-Carvalho et al. (2009, 2011); 20, Condez et al. (2016); 21, Conte \& Machado (2005); 22-23, Conte \& Rossa-Feres (2006, 2007); 24-25, Conte et al. (2005, 2010); 26, Costa et al. (2009); 27, Crivellari et al. (2014); 28, Cruz (1990); 29, Cruz et al. (2008); 30, Cunha et al. (2010); 31, Figueiredo et al. (2014); 32, Fontoura et al. (2011); 33, Gambale et al. (2014); 34, Garey \& Hartmann (2012); 35-36, Garey et al. (2012a, 2012b); 37-38, Heyer (1978, 1983); 39, Heyer \& Heyer (2004); 40, Hiert \& Moura (2010); 41, Hiert et al. (2012); 42, Izecksohn (1993); 43, Langone \& Segalla (1996); 44, Langone et al. (2008); 45, Leivas \& Hiert (2016); 46, Lima et al. (2010); 47, Lingnau \& Bastos (2007); 48, Lingnau et al. (2008); 49, Machado \& Bernarde (2002); 50, Machado et al. (1999); 51, Marcelino et al. (2009); 52, Miranda et al. (2008); 53-55, Moresco et al. (2009, 2013, 2014); 56, Nazaretti \& Conte (2015); 57, Nunes et al. (2012); 58, Oda \& Landgraf (2012); 59, Oda et al. (2015); 60, Oliveira et al. (2010); 61, Pederassi et al. (2015); 62, Pie et al. (2013); 63, Pimenta et al. (2014); 64, Pombal et al. (1998); 65, Prado \& Pombal (2008); 66, Ribeiro et al. (2015); 67, Ribeiro et al. (2017); 68, Sá \& Langone (2002); 69-70, Sá et al. (2007, 2014); 71, SantosPereira \& Rocha (2015); 72-74, Santos-Pereira et al. (2011, 2015, 2016); 75, Steinbach-Padilha (2008); 76-77, Toledo et al. (2007, 2012); 78, Thomé et al. (2012); 79, Trein et al. (2014); 80, Vieira et al. (2012); 81, Winkelmann \& Noleto (2015). *considered the anuran species as "aff.". **considered the anuran species as "cf.".

\begin{tabular}{|c|c|c|c|c|c|c|c|}
\hline \multirow[b]{2}{*}{ TAXON } & \multirow{2}{*}{$\begin{array}{c}\text { Geografic Distribution in } \\
\text { Brazil }\end{array}$} & \multirow[b]{2}{*}{ Endemism } & \multicolumn{3}{|c|}{ Red lists } & \multirow{2}{*}{$\begin{array}{c}\text { Population } \\
\text { trend } \\
\text { (IUCN) }\end{array}$} & \multirow[b]{2}{*}{$\begin{array}{l}\text { Source of } \\
\text { record }\end{array}$} \\
\hline & & & Paraná & Brazil & $\begin{array}{r}\text { Global } \\
(\text { IUCN) }\end{array}$ & & \\
\hline
\end{tabular}

\section{Alsodidae}

Limnomedusa macroglossa

Southern

CR

LC

S

9,22

(Duméril \& Bibron, 1841)

\section{Brachycephalidae}

Brachycephalus brunneus Ribeiro, Alves, Haddad \& Reis, 2005

Brachycephalus coloratus

Ribeiro, Blackburn, Stanley, Pie \&

Bornschein, 2017

Brachycephalus curupira Ribeiro, Blackburn, Stanley, Pie \&

Bornschein, 2017

Brachycephalus ferruginus Alves, Ribeiro, Haddad \& Reis, 2006

Brachycephalus hermogenesi (Giaretta \& Sawaya, 1998)

Brachycephalus izecksohni Ribeiro, Alves, Haddad \& Reis, 2005

Brachycephalus leopardus Ribeiro, Firkowski \& Pie, 2015

Brachycephalus pernix Pombal, Wistuba \& Bornschein, 1998

Brachycephalus pombali Alves, Ribeiro, Haddad \& Reis, 2006

Brachycephalus sulfuratus Condez, Monteiro, Comitti, Garcia, Amaral \& Haddad, 2016

Brachycephalus tridactylus Garey, Lima, Hartmann \& Haddad, 2012

Ischnocnema henselii (Peters, 1870)
PR (Pico Caratuva, Campina Grande do Sul)

PR (Serra da Baitaca, Piraquara)

PR (Serra do Salto, Malhada District, São José dos Pinhais)

PR (Pico Marumbi, Morretes)

RJ, SP and PR

X

$\mathrm{X}$

$\mathrm{X}$

DD

U

$13,18,19$, 32,62

67

67

X

$\mathrm{X}$

PR (Pico Caratuva, Campina Grande do Sul)

PR (Serra do Araçatuba, Tijucas do Sul and Morro dos Perdidos, Guaratuba)

PR (Quatro Barras, Morretes and São José dos Pinhais)

PR (Pico da Igreja, Guaratuba)

SP, PR and SC

PR (Pico do Morato, Guaraqueçaba)

Southern

X

X

X

X

CR

CR $\quad \mathrm{CR}$

DD

U

4, 13, 18

LC

S

$30 *, 72,74$

DD

U

$13,18,19,62$

13,66

$18,19,22$ 62,64

DD

U 4, 13, 18, 19, 62

20

$11,13,34$ 36,74

LC

U

$6^{*}, 8,9,21$, $22,23,25,27$, $30 *, 34,45$, $49,50,72$, 73,74 Ischnocnema paranaensis (Langone \& Segalla, 1996)

PR (Next to Pico do Paraná) X
X EN

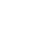

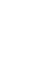

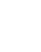

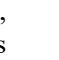

\section{.}

.
.

\section{.} . 
Continued Table 1.

\begin{tabular}{|c|c|c|c|c|c|c|c|}
\hline \multirow{2}{*}{ TAXON } & \multirow{2}{*}{$\begin{array}{c}\text { Geografic Distribution in } \\
\text { Brazil }\end{array}$} & \multirow[b]{2}{*}{ Endemism } & \multicolumn{3}{|c|}{ Red lists } & \multirow{2}{*}{$\begin{array}{c}\text { Population } \\
\text { trend } \\
\text { (IUCN) }\end{array}$} & \multirow{2}{*}{$\begin{array}{l}\text { Source of } \\
\text { record }\end{array}$} \\
\hline & & & Paraná & Brazil & $\begin{array}{l}\text { Global } \\
(\text { IUCN) }\end{array}$ & & \\
\hline $\begin{array}{l}\text { Ischnocnema sambaqui (Castanho } \\
\text { \& Haddad, 2000) }\end{array}$ & $\begin{array}{l}\text { PR (Guaraqueçaba and } \\
\text { Morretes) }\end{array}$ & $\mathrm{X}$ & DD & & $\mathrm{DD}$ & $\mathrm{U}$ & $6,17,22,74$ \\
\hline Ischnocnema cf. spanios & & & & & DD & $\mathrm{U}$ & 74 \\
\hline \multicolumn{8}{|l|}{ Bufonidae } \\
\hline $\begin{array}{l}\text { Dendrophryniscus leucomystax } \\
\text { Izecksohn, } 1968\end{array}$ & RJ, SP, PR and SC & & & & $\mathrm{LC}$ & $\mathrm{D}$ & 34,74 \\
\hline $\begin{array}{l}\text { Dendrophryniscus stawiarskyi } \\
\text { Izecksohn, } 1994\end{array}$ & PR (Bituruna) & $\mathrm{X}$ & DD & & DD & $\mathrm{U}$ & 42 \\
\hline $\begin{array}{l}\text { Melanophryniscus vilavelhensis } \\
\text { Steinback-Padilha, } 2008\end{array}$ & PR (Ponta Grossa) & $X$ & & & & & 27,75 \\
\hline $\begin{array}{l}\text { Rhinella abei (Baldissera, } \\
\text { Caramaschi \& Haddad, 2004) }\end{array}$ & Southern & & & & $\mathrm{LC}$ & $\mathrm{U}$ & $\begin{array}{c}6,21,22,23 \\
27,30,34,45 \\
72,74,78\end{array}$ \\
\hline Rhinella henseli (Lutz, 1934) & Southern & & & & $\mathrm{LC}$ & $\mathrm{U}$ & 27,78 \\
\hline $\begin{array}{l}\text { Rhinella hoogmoedi Caramaschi \& } \\
\text { Pombal, } 2006\end{array}$ & CE to PR & & & & $\mathrm{LC}$ & $\mathrm{U}$ & 34,74 \\
\hline Rhinella icterica (Spix, 1824) & RS to BA, MG and GO & & & & $\mathrm{LC}$ & $\mathrm{S}$ & $\begin{array}{c}6,21,22,23 \\
27,30,34,45 \\
49,74\end{array}$ \\
\hline \multicolumn{8}{|l|}{ Craugastoridae } \\
\hline Haddadus binotatus (Spix, 1824) & BA to RS, MS & & & & $\mathrm{LC}$ & $\mathrm{S}$ & $\begin{array}{c}8,9,22,27 \\
34,49,50 \\
72,74\end{array}$ \\
\hline \multicolumn{8}{|l|}{ Cycloramphidae } \\
\hline Cycloramphus cf. asper & & & DD & & DD & $\mathrm{D}$ & 74 \\
\hline $\begin{array}{l}\text { Cycloramphus bolitoglossus } \\
\text { (Werner, 1897) }\end{array}$ & $\mathrm{PR}$ and $\mathrm{SC}$ & & DD & & DD & $\mathrm{D}$ & $21,22,23,38$ \\
\hline $\begin{array}{l}\text { Cycloramphus duseni (Andersson, } \\
\text { 1914) }\end{array}$ & PR (Ipiranga) & $\mathrm{X}$ & DD & & DD & $\mathrm{U}$ & 38 \\
\hline $\begin{array}{l}\text { Cycloramphus eleutherodactylus } \\
\text { (Miranda-Ribeiro, 1920) }\end{array}$ & RJ, SP and PR & & DD & & DD & $\mathrm{U}$ & 38 \\
\hline $\begin{array}{l}\text { Cycloramphus lutzorum Heyer, } \\
1983\end{array}$ & SP and PR & & DD & & DD & $\mathrm{D}$ & 38,46 \\
\hline $\begin{array}{l}\text { Cycloramphus mirandaribeiroi } \\
\text { Heyer, } 1983\end{array}$ & PR (São João da Graciosa) & $X$ & DD & & DD & $\mathrm{U}$ & 38,74 \\
\hline
\end{tabular}


Continued Table 1.

\begin{tabular}{|c|c|c|c|c|c|c|c|}
\hline \multirow[b]{2}{*}{ TAXON } & \multirow{2}{*}{$\begin{array}{c}\text { Geografic Distribution in } \\
\text { Brazil }\end{array}$} & \multirow[b]{2}{*}{ Endemism } & \multicolumn{3}{|c|}{ Red lists } & \multirow{2}{*}{$\begin{array}{c}\text { Population } \\
\text { trend } \\
\text { (IUCN) }\end{array}$} & \multirow{2}{*}{$\begin{array}{l}\text { Source of } \\
\text { record }\end{array}$} \\
\hline & & & Paraná & Brazil & $\begin{array}{l}\text { Global } \\
\text { (IUCN) }\end{array}$ & & \\
\hline $\begin{array}{l}\text { Cycloramphus rhyakonastes Heyer, } \\
1983\end{array}$ & $\mathrm{PR}$ and $\mathrm{SC}$ & & $\mathrm{DD}$ & & $\mathrm{LC}$ & $\mathrm{U}$ & 38 \\
\hline \multicolumn{8}{|l|}{ Hemiphractidae } \\
\hline $\begin{array}{l}\text { Gastrotheca microdiscus } \\
\text { (Andersson, 1910) }\end{array}$ & $\mathrm{SP}, \mathrm{PR}$ and $\mathrm{SC}$ & & DD & & $\mathrm{LC}$ & $\mathrm{D}$ & 15,74 \\
\hline Fritziana gr. fissilis & & & & & $\mathrm{LC}$ & $\mathrm{S}$ & 34,74 \\
\hline \multicolumn{8}{|l|}{ Hylidae } \\
\hline $\begin{array}{l}\text { Aplastodiscus albosignatus (Lutz \& } \\
\text { Lutz, 1938) }\end{array}$ & GO, MG, RJ, SP and PR & & & & $\mathrm{LC}$ & $\mathrm{D}$ & $\begin{array}{l}21,22,23,27 \\
30,45,74\end{array}$ \\
\hline $\begin{array}{l}\text { Aplastodiscus ehrhardti (Müller, } \\
\text { 1924) }\end{array}$ & $\mathrm{SP}, \mathrm{PR}$ and $\mathrm{SC}$ & & & & $\mathrm{LC}$ & $\mathrm{D}$ & $6,22,24$ \\
\hline Boana albomarginata (Spix, 1824) & $\mathrm{PE}$ to $\mathrm{SC}$ & & & & $\mathrm{LC}$ & $\mathrm{S}$ & $6,30,34,74$ \\
\hline Boana albopunctata (Spix, 1824) & $\begin{array}{l}\text { Central, Southeastern and } \\
\text { Southern }\end{array}$ & & & & $\mathrm{LC}$ & S & $\begin{array}{c}1,3,8,21,22 \\
27,45,49,50 \\
56,75\end{array}$ \\
\hline Boana bischoffi (Boulenger, 1887) & $\mathrm{RJ}$ to $\mathrm{RS}$ & & & & $\mathrm{LC}$ & S & $\begin{array}{l}6,21,22,23 \\
27,30,45,51\end{array}$ \\
\hline Boana caingua (Carrizo, 1991) & MS, SP and Southern & & & & $\mathrm{LC}$ & $\mathrm{S}$ & 27 \\
\hline Boana faber (Wied-Neuwied, 1821) & PE to RS & & & & $\mathrm{LC}$ & $\mathrm{S}$ & $\begin{array}{c}1,3,6,8,9 \\
21,22,23,27, \\
28,34,45,49, \\
50,55,74,80\end{array}$ \\
\hline Boana prasina (Burmeister, 1856) & MG, RJ, SP and PR & & & & $\mathrm{LC}$ & $\mathrm{S}$ & $\begin{array}{c}8,9,21,22 \\
23,27,45,49 \\
50,56\end{array}$ \\
\hline $\begin{array}{l}\text { Boana pulchella (Duméril \& } \\
\text { Bibron, 1841) }\end{array}$ & Southern & & & & $\mathrm{LC}$ & $\mathrm{S}$ & 27 \\
\hline Boana raniceps (Cope, 1862) & $\begin{array}{c}\text { Southeastern (except ES) } \\
\text { and PR }\end{array}$ & & & & $\mathrm{LC}$ & $\mathrm{S}$ & $\begin{array}{l}1,3,22,33 \\
49,50,56\end{array}$ \\
\hline Boana semiguttata (Lutz, 1925) & $\mathrm{PR}$ and $\mathrm{SC}$ & & & EN & $\mathrm{LC}$ & $\mathrm{S}$ & 22,49 \\
\hline Boana semilineata (Spix, 1824) & $\mathrm{AL}$ to $\mathrm{SC}$ & & & & $\mathrm{LC}$ & $\mathrm{S}$ & 34,74 \\
\hline Boana cf. stellae & & & & & $\mathrm{LC}$ & $\mathrm{S}$ & 27 \\
\hline $\begin{array}{l}\text { Bokermannohyla circumdata } \\
\text { (Cope, 1871) }\end{array}$ & $\begin{array}{c}\text { BA, Southeastern, PR and } \\
\text { SC }\end{array}$ & & & & $\mathrm{LC}$ & $\mathrm{D}$ & $21,22,23,27$ \\
\hline $\begin{array}{l}\text { Bokermannohyla hylax (Heyer, } \\
\text { 1985) }\end{array}$ & SP and PR & & & & $\mathrm{LC}$ & $\mathrm{D}$ & $6,30,34,74$ \\
\hline $\begin{array}{l}\text { Bokermannohyla langei } \\
\text { (Bokermann, 1965) }\end{array}$ & PR (Morretes) & $X$ & DD & & DD & $\mathrm{U}$ & 10 \\
\hline $\begin{array}{l}\text { Dendropsophus anceps (Lutz, } \\
\text { 1929) }\end{array}$ & BA to PR & & $\mathrm{CR}$ & & $\mathrm{LC}$ & $\mathrm{S}$ & $22,25,56$ \\
\hline
\end{tabular}


Continued Table 1.

\begin{tabular}{|c|c|c|c|c|c|c|c|}
\hline \multirow[b]{2}{*}{ TAXON } & \multirow{2}{*}{$\begin{array}{c}\text { Geografic Distribution in } \\
\text { Brazil }\end{array}$} & \multirow[b]{2}{*}{ Endemism } & \multicolumn{3}{|c|}{ Red lists } & \multirow{2}{*}{$\begin{array}{l}\text { Population } \\
\text { trend } \\
\text { (IUCN) }\end{array}$} & \multirow{2}{*}{$\begin{array}{l}\text { Source of } \\
\text { record }\end{array}$} \\
\hline & & & Paraná & Brazil & $\begin{array}{r}\text { Global } \\
(\text { IUCN) }\end{array}$ & & \\
\hline $\begin{array}{l}\text { Dendropsophus berthalutzae } \\
\text { (Bokermann, 1962) }\end{array}$ & ES, SP and PR & & & & $\mathrm{LC}$ & $\mathrm{S}$ & $6,30,34,74$ \\
\hline $\begin{array}{l}\text { Dendropsophus elegans (Wied- } \\
\text { Neuwied, 1824) }\end{array}$ & $\mathrm{BA}$ to $\mathrm{SC}$ & & & & $\mathrm{LC}$ & $\mathrm{S}$ & $6,30,34,74$ \\
\hline $\begin{array}{l}\text { Dendropsophus minutus (Peters, } \\
\text { 1872) }\end{array}$ & Brazil & & & & $\mathrm{LC}$ & $\mathrm{S}$ & $\begin{array}{l}1,3,6,8,9, \\
21,22,23,27, \\
30,34,45,49, \\
50,56,74,75\end{array}$ \\
\hline $\begin{array}{l}\text { Dendropsophus nahdereri (Lutz \& } \\
\text { Bokermann, 1963) }\end{array}$ & Southern & & & & $\mathrm{LC}$ & $\mathrm{S}$ & $22,25,30$ \\
\hline $\begin{array}{l}\text { Dendropsophus sanborni (Schmidt, } \\
\text { 1944) }\end{array}$ & Southern & & & & $\mathrm{LC}$ & $\mathrm{S}$ & $\begin{array}{c}21,22,23,27, \\
49,56\end{array}$ \\
\hline $\begin{array}{l}\text { Dendropsophus seniculus (Cope, } \\
\text { 1868) }\end{array}$ & MG, ES, RJ and PR & & & & $\mathrm{LC}$ & $\mathrm{S}$ & 34,74 \\
\hline $\begin{array}{l}\text { Dendropsophus werneri (Cochran, } \\
\text { 1952) }\end{array}$ & Southern & & & & $\mathrm{LC}$ & $\mathrm{S}$ & $\begin{array}{l}30,34,52 \\
\quad 60,74\end{array}$ \\
\hline $\begin{array}{l}\text { Itapothihyla langsdorffii (Duméril } \\
\& \text { Bibron, 1841) }\end{array}$ & $\begin{array}{l}\text { SE, BA, Southeastern, PR } \\
\text { and RS }\end{array}$ & & & & & & 74 \\
\hline $\begin{array}{l}\text { Julianus uruguayus (Schmidt, } \\
\text { 1944) }\end{array}$ & $\mathrm{PR}$ and $\mathrm{SC}$ & & & & $\mathrm{LC}$ & $\mathrm{S}$ & $22,27,49$ \\
\hline $\begin{array}{l}\text { Ololygon catharinae (Boulenger, } \\
\text { 1888) }\end{array}$ & Southeastern and Southern & & & & $\mathrm{LC}$ & $\mathrm{S}$ & $22,23,27 * *$ \\
\hline $\begin{array}{l}\text { Ololygon littoralis (Pombal \& } \\
\text { Gordo, 1991) }\end{array}$ & SP, PR and SC & & & & $\mathrm{LC}$ & $\mathrm{D}$ & $\begin{array}{c}6,34,35,74 \\
77\end{array}$ \\
\hline Ololygon aff. perpusilla & & & & & $\mathrm{LC}$ & $\mathrm{S}$ & $6,34,74$ \\
\hline $\begin{array}{l}\text { Ololygon rizibilis (Bokermann, } \\
\text { 1964) }\end{array}$ & SP and PR & & & & $\mathrm{LC}$ & $\mathrm{D}$ & $\begin{array}{c}6,22,23,27 \\
30,31\end{array}$ \\
\hline Phasmahyla guttata (Lutz, 1924) & RJ, SP, ES and PR & & DD & & $\mathrm{LC}$ & $\mathrm{D}$ & 28 \\
\hline Phyllomedusa distincta Lutz, 1950 & $\mathrm{SP}, \mathrm{PR}$ and $\mathrm{SC}$ & & & & $\mathrm{LC}$ & $\mathrm{D}$ & $\begin{array}{l}6,22,23,27 \\
30,34,45,74\end{array}$ \\
\hline $\begin{array}{l}\text { Phyllomedusa tetraploidea Pombal } \\
\text { \& Haddad, } 1992\end{array}$ & SP and PR & & & & $\mathrm{LC}$ & $\mathrm{S}$ & $\begin{array}{c}1,3,8,9,22 \\
27,49,50 \\
56,81\end{array}$ \\
\hline $\begin{array}{l}\text { Pithecopus rusticus (Bruschi, } \\
\text { Lucas, Garcia \& Recco-Pimentel, } \\
\text { 2015) }\end{array}$ & $\mathrm{PR}$ and $\mathrm{SC}$ & & & & & & 27 \\
\hline Pseudis cardosoi Kwet, 2000 & Southern & & & & $\mathrm{LC}$ & $\mathrm{S}$ & 25,27 \\
\hline
\end{tabular}


Continued Table 1.

\begin{tabular}{|c|c|c|c|c|c|c|c|}
\hline \multirow[b]{2}{*}{ TAXON } & \multirow[b]{2}{*}{$\begin{array}{c}\text { Geografic Distribution in } \\
\text { Brazil }\end{array}$} & \multirow[b]{2}{*}{ Endemism } & \multicolumn{3}{|c|}{ Red lists } & \multirow{2}{*}{$\begin{array}{l}\text { Population } \\
\text { trend } \\
\text { (IUCN) }\end{array}$} & \multirow[b]{2}{*}{$\begin{array}{l}\text { Source of } \\
\text { record }\end{array}$} \\
\hline & & & Paraná & Brazil & $\begin{array}{l}\text { Global } \\
\text { (IUCN) }\end{array}$ & & \\
\hline Scinax alter (Lutz, 1973) & $\mathrm{BA}, \mathrm{MG}, \mathrm{ES}, \mathrm{RJ}$ and PR & & & & $\mathrm{LC}$ & $\mathrm{S}$ & $6^{*}, 30$ \\
\hline $\begin{array}{l}\text { Scinax fuscomarginatus (Lutz, } \\
\text { 1925) }\end{array}$ & $\begin{array}{c}\text { Southern, Central, } \\
\text { Southeastern, Northeast, } \\
\text { Southern AM }\end{array}$ & & & & $\mathrm{LC}$ & S & 56 \\
\hline Scinax granulatus (Peters, 1871) & Southern & & & & $\mathrm{LC}$ & $\mathrm{S}$ & $25,27,53$ \\
\hline $\begin{array}{l}\text { Scinax imbegue Nunes, Kwet \& } \\
\text { Pombal, } 2012\end{array}$ & SP, PR and SC & & & & & & 57 \\
\hline Scinax squalirostris (Lutz, 1925) & MS, Southeastern and PR & & & & $\mathrm{LC}$ & $\mathrm{S}$ & $21,22,27,49$ \\
\hline $\begin{array}{l}\text { Scinax tymbamirim Nunes, Kwet \& } \\
\text { Pombal, } 2012\end{array}$ & $\mathrm{RJ}$ to $\mathrm{RS}$ & & & & & & $57,74 * *$ \\
\hline $\begin{array}{l}\text { Sphaenorhynchus caramaschii } \\
\text { Toledo, Garcia, Lingnau \& Haddad, } \\
2007\end{array}$ & $\begin{array}{l}\text { Southern, Southeastern and } \\
\text { BA }\end{array}$ & & & & $\mathrm{LC}$ & S & 27,76 \\
\hline $\begin{array}{l}\text { Sphaenorhynchus surdus (Cochran, } \\
\text { 1953) }\end{array}$ & Southern & & & & $\mathrm{LC}$ & $\mathrm{S}$ & $21,22,23,27$ \\
\hline \multicolumn{8}{|l|}{ Hylodidae } \\
\hline $\begin{array}{l}\text { Crossodactylus caramaschii Bastos } \\
\text { \& Pombal, } 1995\end{array}$ & SP, PR and SC & & & & $\mathrm{LC}$ & $\mathrm{S}$ & $27^{* *}, 63,74$ \\
\hline $\begin{array}{l}\text { Crossodactylus schmidti Gallardo, } \\
1961\end{array}$ & Southern & & & & NT & $\mathrm{D}$ & 3 \\
\hline $\begin{array}{l}\text { Hylodes cardosoi Lingnau, Canedo } \\
\text { \& Pombal, } 2008\end{array}$ & SP and PR & & & & $\mathrm{LC}$ & $\mathrm{U}$ & $48,74,80$ \\
\hline Hylodes aff. asper & & & & & $\mathrm{LC}$ & $\mathrm{S}$ & 34,74 \\
\hline $\begin{array}{l}\text { Hylodes heyeri Haddad, Pombal \& } \\
\text { Bastos, } 1996\end{array}$ & SP and PR & & & & DD & $\mathrm{D}$ & $\begin{array}{l}6,22,26,30 \\
34,47,74 * *\end{array}$ \\
\hline \multicolumn{8}{|l|}{ Leptodactylidae } \\
\hline $\begin{array}{l}\text { Adenomera araucaria Kwet \& } \\
\text { Angulo, } 2002\end{array}$ & Southern & & & & $\mathrm{LC}$ & $\mathrm{S}$ & 25 \\
\hline Adenomera cf. bokermanni & & & & & $\mathrm{LC}$ & $\mathrm{S}$ & $6^{*}, 34^{*}, 74^{* *}$ \\
\hline $\begin{array}{l}\text { Adenomera marmorata } \\
\text { Steindachner, } 1867\end{array}$ & $\mathrm{RJ}$ to $\mathrm{SC}$ & & & & $\mathrm{LC}$ & S & $\begin{array}{c}21,22,23 * \\
27^{*}, 30^{* *}, 34 \\
\quad 72,73,74\end{array}$ \\
\hline
\end{tabular}


Continued Table 1.

\begin{tabular}{|c|c|c|c|c|c|c|c|}
\hline \multirow[b]{2}{*}{ TAXON } & \multirow{2}{*}{$\begin{array}{c}\text { Geografic Distribution in } \\
\text { Brazil }\end{array}$} & \multirow[b]{2}{*}{ Endemism } & \multicolumn{3}{|c|}{ Red lists } & \multirow{2}{*}{$\begin{array}{c}\text { Population } \\
\text { trend } \\
\text { (IUCN) }\end{array}$} & \multirow{2}{*}{$\begin{array}{l}\text { Source of } \\
\text { record }\end{array}$} \\
\hline & & & Paraná & Brazil & $\begin{array}{l}\text { Global } \\
(\text { IUCN) }\end{array}$ & & \\
\hline Adenomera nana (Müller, 1922) & $\mathrm{PR}$ and $\mathrm{SC}$ & & & & $\mathrm{LC}$ & $\mathrm{U}$ & 25,27 \\
\hline $\begin{array}{l}\text { Leptodactylus flavopictus Lutz, } \\
1926\end{array}$ & $\mathrm{ES}$ to $\mathrm{SC}$ & & & & $\mathrm{LC}$ & $\mathrm{D}$ & 70 \\
\hline $\begin{array}{l}\text { Leptodactylus furnarius Sazima \& } \\
\text { Bokermann, } 1978\end{array}$ & $\begin{array}{c}\text { TO, BA, Central, } \\
\text { Southeastern and PR }\end{array}$ & & & & $\mathrm{LC}$ & $\mathrm{S}$ & $7,37,39,70$ \\
\hline $\begin{array}{l}\text { Leptodactylus fuscus } \\
\text { (Schneider,1799) }\end{array}$ & Brazil & & & & $\mathrm{LC}$ & $\mathrm{S}$ & $\begin{array}{c}1,3,8,22,27 \\
33,49,50 \\
56,70\end{array}$ \\
\hline $\begin{array}{l}\text { Leptodactylus gracilis (Duméril \& } \\
\text { Bibron, 1840) }\end{array}$ & Southern & & & & $\mathrm{LC}$ & $\mathrm{S}$ & $22,27,49,70$ \\
\hline $\begin{array}{l}\text { Leptodactylus mystaceus (Spix, } \\
\text { 1824) }\end{array}$ & $\begin{array}{l}\text { Norte, Northeast, Central, } \\
\text { MG, SP and PR }\end{array}$ & & & & $\mathrm{LC}$ & $\mathrm{S}$ & $2,3,70$ \\
\hline $\begin{array}{l}\text { Leptodactylus mystacinus } \\
\text { (Burmeister, 1861) }\end{array}$ & $\begin{array}{l}\text { Central, Southeastern and } \\
\text { Southern }\end{array}$ & & & & $\mathrm{LC}$ & $\mathrm{S}$ & $\begin{array}{l}1,3,9,22,27 \\
49,50,55,70\end{array}$ \\
\hline $\begin{array}{l}\text { Leptodactylus notoaktites Heyer, } \\
1978\end{array}$ & $\mathrm{SP}, \mathrm{PR}$ and $\mathrm{SC}$ & & & & $\mathrm{LC}$ & $\mathrm{S}$ & $\begin{array}{c}6,22,23,27 \\
30,34,45,69 \\
70,74\end{array}$ \\
\hline Leptodactylus plaumanni Ahl, 1936 & Southern & & & & $\mathrm{LC}$ & $\mathrm{S}$ & 27,70 \\
\hline $\begin{array}{l}\text { Leptodactylus podicipinus (Cope, } \\
\text { 1862) }\end{array}$ & Northern, Central and PR & & DD & & $\mathrm{LC}$ & $\mathrm{S}$ & $\begin{array}{c}3,22,49,56 \\
70\end{array}$ \\
\hline $\begin{array}{l}\text { Physalaemus insperatus Cruz, } \\
\text { Cassini \& Caramaschi, } 2008\end{array}$ & PR (Guaratuba) & $\mathrm{X}$ & & & DD & $\mathrm{U}$ & 29 \\
\hline $\begin{array}{l}\text { Physalaemus lateristriga } \\
\text { (Steindachner, 1864) }\end{array}$ & $\mathrm{SP}, \mathrm{PR}$ and $\mathrm{SC}$ & & & & & & 27,45 \\
\hline $\begin{array}{l}\text { Physalaemus maculiventris (Lutz, } \\
\text { 1925) }\end{array}$ & RJ, SP, PR and SC & & DD & & $\mathrm{LC}$ & $\mathrm{D}$ & 30 \\
\hline $\begin{array}{l}\text { Physalaemus nanus (Boulenger, } \\
\text { 1888) }\end{array}$ & SP and Southern & & & & $\mathrm{LC}$ & $\mathrm{S}$ & 27 \\
\hline $\begin{array}{l}\text { Physalaemus nattereri } \\
\text { (Steindachner, 1863) }\end{array}$ & Central, Southeastern and SC & & & & $\mathrm{LC}$ & $\mathrm{D}$ & 3,56 \\
\hline $\begin{array}{l}\text { Physalaemus olfersii (Lichtenstein } \\
\text { \& Martens, 1856) }\end{array}$ & MG, ES, SP and PR & & & & $\mathrm{LC}$ & $\mathrm{S}$ & $\begin{array}{l}6^{* *}, 22,23 \\
30,34^{*}, 74^{*}\end{array}$ \\
\hline $\begin{array}{l}\text { Physalaemus spiniger (Miranda- } \\
\text { Ribeiro, 1926) }\end{array}$ & SP and PR & & & & $\mathrm{LC}$ & $\mathrm{D}$ & $34,72,74,77$ \\
\hline Pleurodema bibroni Tschudi, 1838 & Southern & & & & NT & $\mathrm{D}$ & $27^{* *}, 79$ \\
\hline $\begin{array}{l}\text { Scythrophrys sawayae (Cochran, } \\
\text { 1953) }\end{array}$ & $\mathrm{PR}$ and $\mathrm{SC}$ & & DD & & $\mathrm{LC}$ & $\mathrm{D}$ & 22 \\
\hline
\end{tabular}


Continued Table 1.

\begin{tabular}{|c|c|c|c|c|c|c|c|}
\hline \multirow{2}{*}{ TAXON } & \multirow{2}{*}{$\begin{array}{c}\text { Geografic Distribution in } \\
\text { Brazil }\end{array}$} & \multirow[b]{2}{*}{ Endemism } & \multicolumn{3}{|c|}{ Red lists } & \multirow{2}{*}{$\begin{array}{c}\text { Population } \\
\text { trend } \\
\text { (IUCN) }\end{array}$} & \multirow{2}{*}{$\begin{array}{l}\text { Source of } \\
\text { record }\end{array}$} \\
\hline & & & Paraná & Brazil & $\begin{array}{r}\text { Global } \\
\text { (IUCN) }\end{array}$ & & \\
\hline \multicolumn{8}{|l|}{ Microhylidae } \\
\hline $\begin{array}{l}\text { Chiasmocleis leucosticta } \\
\text { (Boulenger, 1888) }\end{array}$ & $\mathrm{SP}, \mathrm{PR}$ and $\mathrm{SC}$ & & $\mathrm{DD}$ & & $\mathrm{LC}$ & $\mathrm{S}$ & 22,30 \\
\hline $\begin{array}{l}\text { Elachistocleis bicolor (Guérin- } \\
\text { Méneville, 1838) }\end{array}$ & Southern & & & & $\mathrm{LC}$ & $\mathrm{S}$ & $\begin{array}{c}3 * *, 6,22,23 \\
50,56\end{array}$ \\
\hline Elachistocleis cf. cesarii & & & & & & & 1 \\
\hline \multicolumn{8}{|l|}{ Odontophrynidae } \\
\hline $\begin{array}{l}\text { Odontophrynus americanus } \\
\text { (Duméril \& Bibron, 1841) }\end{array}$ & Southern & & & & $\mathrm{LC}$ & S & $\begin{array}{l}3,21,22,23 \\
27,49,50,56\end{array}$ \\
\hline $\begin{array}{l}\text { Proceratophrys avelinoi Mercadal } \\
\text { de Barrio \& Barrio, } 1993\end{array}$ & $\mathrm{PR}$ and $\mathrm{RS}$ & & & & $\mathrm{LC}$ & $\mathrm{U}$ & $\begin{array}{l}8,9,22,23^{*} \\
49,50,68\end{array}$ \\
\hline $\begin{array}{l}\text { Proceratophrys boiei (Wied- } \\
\text { Neuwied, 1824) }\end{array}$ & Southeastern, PR and SC & & & & $\mathrm{LC}$ & $\mathrm{S}$ & $\begin{array}{c}6,21,22,23 \\
27,30,34,45 \\
72,74\end{array}$ \\
\hline $\begin{array}{l}\text { Proceratophrys subguttata } \\
\text { Izecksohn, Cruz \& Peixoto, } 1999\end{array}$ & $\mathrm{PR}$ and $\mathrm{SC}$ & & & & $\mathrm{LC}$ & $\mathrm{D}$ & 65 \\
\hline \multicolumn{8}{|l|}{ Ranidae } \\
\hline $\begin{array}{l}\text { Lithobates catesbeianus (Shaw, } \\
1802 \text { ) }\end{array}$ & Brazil & & & & $\mathrm{LC}$ & I & $\begin{array}{l}1,3,6,9,22 \\
27,45,49,71\end{array}$ \\
\hline
\end{tabular}

We recorded a total of 24 anuran species whose type locality is in the state of Paraná (Figure 1). Nineteen of these species are endemic to the state, and 15 (except B. leopardus, B. pernix, I. sambaqui, and $B$. jaguariavensis) are known only from the type locality (Figure 1).

We also reported the occurrence of the exotic invader anuran Lithobates catesbeianus (Shaw 1802) (Ranidae), known as the bullfrog. We found five studies that recorded the occurrence of this anuran species in the state of Paraná (Table 1).

Five (3.6\%) anuran species are listed as threatened in the red lists of Paraná, Brazil and/or IUCN (Table 1): Brachycephalus pernix, Dendropsophus anceps (Lutz, 1929), Limnomedusa macroglossa (Duméril \& Bibron, 1841), Boana semiguttata (Lutz, 1925) and Ischnocnema paranaensis. Brachycephalus pernix is classified as Critically Endangered (CR) in Paraná and Brazil and L. macroglossa only in Paraná, while B. semiguttata and I. paranaensis are listed as Endangered (EN) in Brazil and in Paraná, respectively. Moreover, Crossodactylus schmidti Gallardo, 1961 and Pleurodema bibroni Tschudi, 1838 are listed as Near Threatened (NT) by the IUCN. A further 27 species (19.2\% of the total) are listed as Data Deficient (DD) in Paraná and/or by the IUCN. Two of the species endemic to Paraná (B. pernix and I. paranaensis) are listed as Critically Endangered, corresponding to $10.5 \%$ of the anuran species endemic to the state.

Based on IUCN (2017), the population trends of 68 (49.6\%) anuran species recorded in the present study are stable (Table 1). Most of these species belongs to the family Hylidae (40 species, $58.8 \%$ of the total number of registered species), representing $69 \%$ of the recorded hylids, and Leptodactylidae (17 species, $25.0 \%$ of the total number of registered species), representing $65.4 \%$ of the recorded leptodactylids. By contrast, 26 species (19\% of the total number of registered species) have declining populations, including nine hylids ( $15.5 \%$ of hylid species) and five leptodactylids (19.2\% of leptodactylids species), and two species have increasing populations (1.4\% of the total number of registered species), Rhinella schneideri (Werner, 1894) and Lithobates catesbeianus. A further 28 (20.4\%) species recorded for Paraná have unknown population trends, nine species from the family Brachycephalidae $(6.6 \%$ of the total number of registered species, $60 \%$ of the brachycephalids) and seven species from the family Bufonidae ( $5.1 \%$ of the total number of registered species, $58.3 \%$ of the bufonids). The remaining 11 anuran species $(8.1 \%$ of the total number of registered species) have yet to be assessed by the IUCN. Two recently described anuran species (Brachycephalus coloratus and B. curupira) have not yet been assessed by the IUCN and therefore, we have not been able to access their population trends.

In our supplementary search of the SpeciesLink database (Table 2), we recorded 24 anuran species belonging to seven families: Hylidae ( $\mathrm{n}=9$ species), Leptodactylidae (5), Hylodidae (4), Bufonidae (2), Centrolenidae (2), Allobatidae (1) and Mycrohylidae (1). These species included 10 anurans not identified in our literature search: Allobates brunneus (Cope, 1887), Vitreorana eurygnata (Lutz, 1925), Aplastodiscus cochranae (Mertens, 1952), A. leucopygius (Cruz \& Peixoto, 1985), Boana polytaenia (Cope, 1870), Ololygon brieni (De Witte, 1930), Crossodactylus gaudichaudii Duméril \& Bibron, 1841, Leptodactylus chaquensis Cei, 1950, Physalaemus biligonigerus (Cope, 1861) and Pseudopaludicola falcipes (Hensel, 1867). 


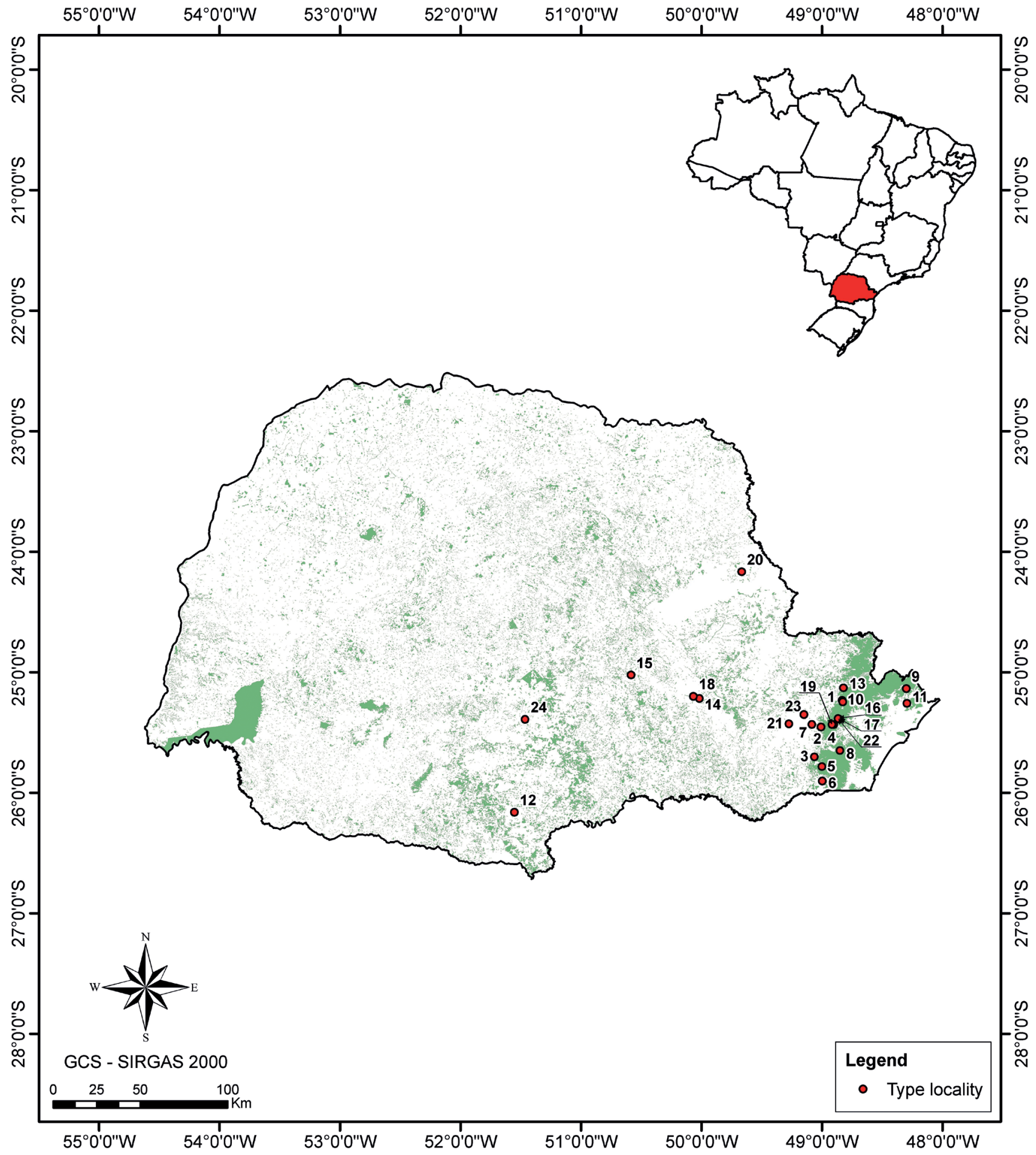

Figure 1. Type localities of the anuran species in the state of Paraná, southern Brazil. Abbreviations: mun., municipality; P.E., Parque Estadual (State Park). Brachycephalus brunneus $(1=$ Pico Caratuva, mun. Campina Grande do Sul), B. ferruginus $(2=$ Pico Marumbi, mun. Morretes $), B$. izecksohni $(3=$ Pico Torre da Prata, from mun. Guaratuba to mun. Paranaguá), B. leopardus (4 = Serra do Araçatuba, mun. Tijucas do Sul), B. pernix (5 = Morro Anhangava, in Serra da Baitaca, Conjunto Marumbi, mun. Quatro Barras), B. pombali $(6=$ Morro dos Padres, Pico da Igreja, mun. Guaratuba $), B$. tridactylus $(7=$ Pico do Morato, mun. Guaraqueçaba), Ischnocnema paranaensis $(8=$ Pico Paraná, mun. Antonina), I. sambaqui $(9$ = mun. Guaraqueçaba), Dendrophryniscus stawiarskyi $(10=$ mun. Bituruna), Melanophryniscus alipioi $(11=$ Serra do Capivarí, mun. Campina Grande do Sul), M. vilavelhensis $(12=\mathrm{P}$. E. de Vila Velha, mun. Ponta Grossa), Cycloramphus duseni $(13$ = mun. Ipiranga), C. mirandaribeiroi $(14=$ São João da Graciosa, mun. Morretes), C. rhyakonastes (15 = São João da Graciosa, mun. Morretes), Gastrotheca microdiscus (16 = mun. Ponta Grossa), Bokermannohyla langei $(17=$ Pico do Marumbi, mun. Morretes), Boana jaguariaivensis $(18=$ P. E. do Cerrado, mun. Jaguariaíva), Sphaenorhynchus surdus $(19=$ mun. Curitiba $)$, Hylodes cardosoi $(20=$ Porto de Cima, mun. Morretes), Physalaemus insperatus (21 = Área de Proteção Ambiental Guaratuba, Serra da Pedra Branca do Araraquara, mun. Guaratuba) and Scythrophrys sawayae (22 = Banhado, mun. Guarapuava). 
Table 2. Putative determinations of the anuran amphibians of the state of Paraná, southern Brazil, that have been deposited in scientific collections (search in the website SpeciesLink 2017). Scientific collections: DZSJRP = Departamento de Zoologia São José do Rio Preto, NMNH = National Museum of Natural History, MZUEL = Museu de Zoologia da Universidade Estadual de Londrina, ZUEC = Museu de Zoologia da Universidade Estadual de Campinas, FNJV $=$ Fonoteca Neotropical Jacques Vielliard, CFBH $=$ Coleção Célio F. B. Haddad. Source: ${ }^{1}=$ Berneck et al. (2016), ${ }^{2}=$ Garcia et al. $(2007),{ }^{3}=$ Lingnau \& Bastos $(2007)$ and ${ }^{4}=$ Sá et al. (2014)

\begin{tabular}{l} 
Taxon \\
\hline Colostethus brunneus (Cope, 1887) \\
Dendrophryniscus brevipollicatus (Jiménez de la \\
Espada, 1870)
\end{tabular}

Bufo crucifer Wied-Neuwied, 1821

Vitreorana eurygnatha (Lutz, 1925)

Vitreorana parvula (Boulenger, 1895)

Aplastodiscus albofrenatus (Lutz, 1924)

Aplastodiscus cochranae (Mertens, 1952)

Aplastodiscus leucopygius (Cruz \& Peixoto, 1985)

Boana joaquini (Lutz, 1968)

Boana polytaenia (Cope, 1870)

Bokermannohyla astartea (Bokermann, 1967)

Ololygon angrensis (Lutz, 1973)

Phrynohyas venulosa (Fitzinger, 1843)

Scinax brieni Faivovich, Haddad, Garcia, Frost \& Wheeler, 2005

Crossodactylus bresslaui Müller, 1924

Crossodactylus gaudichaudii Duméril \& Bibron, 1841

Hylodes asper (Müller, 1924)

Hylodes perplicatus (Miranda-Ribeiroi, 1926)

Leptodactylus bolivianus Boulenger, 1898

Leptodactylus ocellatus Girard, 1853

Physalaemus biligonigerus (Cope, 1861)

Physalaemus marmoratus (Reinhardt \& Lütken, 1862)

Pseudopaludicola falcipes (Hensel, 1867)

Elachistocleis ovalis (Schneider, 1799)
Tentatively updated taxon

Allobates brunneus (Cope, 1887)

Dendrophryniscus stawiarskyi Izecksohn, 1994

Rhinella abei (Baldissera, Caramaschi \& Haddad, 2004)

Vitreorana eurygnatha (Lutz, 1925)

Vitreorana uranoscopa (Müller, 1924)

Aplastodiscus ehrhardti (Müller, 1924) ${ }^{1}$

Aplastodiscus cochranae (Mertens, 1952)

Aplastodiscus leucopygius (Cruz \& Peixoto, 1985)

Boana semiguttata (A. Lutz, 1925) ${ }^{2}$

Boana polytaenia (Cope, 1870)

Bokermannohyla hylax (Heyer, 1985)

Ololygon littoralis (Pombal \& Gordo, 1991)

Trachycephalus typhonius (Linnaeus, 1758)

Ololygon brieni (De Witte, 1930)

Crossodactylus caramaschii Bastos \& Pombal, 1995

Crossodactylus gaudichaudii Duméril \& Bibron, 1841

Hylodes cardosoi Lingnau, Canedo \& Pombal, 2008 or

Hylodes heyeri Haddad, Pombal \& Bastos, $1996^{3}$

Hylodes cardosoi Lingnau, Canedo \& Pombal, 2008 or

Hylodes heyeri Haddad, Pombal \& Bastos, $1996^{3}$

Leptodactylus chaquensis Cei, $1950{ }^{4}$

Leptodactylus latrans (Steffen, 1815)

Physalaemus biligonigerus (Cope, 1861)

Physalaemus marmoratus (Reinhardt \& Lütken, 1862)

Pseudopaludicola falcipes (Hensel, 1867)

Elachistoclei bicolor (Guérin-Méneville, 1838)
Voucher number

NMNH-Animalia_BR 148487

NMNH-Animalia BR 217659

MZUEL-Herpeto 1134

NMNH-Animalia_BR 284502

ZUEC-AMP 284502

DZSJRP 5252

FNJV 0033953

FNJV 0033943

FNJV 0033075

ZUEC-AMP 10502

NMNH 125516

ZUEC-AMP 4724

MZUEL-Herpeto 362

NMNH-Animalia_BR 125515

MZUEL-Herpeto 748

DZSJRP-Amphibia-adults 6296

NMNH-Animalia_BR 149648

NMNH-Animalia BR 125509

DZSJRP-Amphibia-adults 8625 CFBH 21025

ZUEC-AMP 10371

DZSJRP-Amphibia-tadpoles 0832.03

NMNH-Animalia BR 149646

MZUEL-Herpeto 671
We identified 29 localities at which anurans have been inventoried in the state of Paraná, of which, just under half (48.3\%) are located with the metropolitan region of Curitiba city, while a further $34.5 \%$ are found within the central-northern region of the state (Figure 2), creating a highly disproportionate distribution of sampling sites within the state.

\section{Discussion}

The 137 anuran species recorded in the present study for the Brazilian state of Parana is close to the 147 species predicted for the state by Toledo \& Batista (2012), and thus appears to be a relatively reliable estimate. In that study, the authors constructed a Brazilian list of species based on range distribution shapes (shadow maps analysis), including species occurrences by Brazilian states, and therefore, we assume that species recorded by them to Paraná but not registered in our study do not occur in the state; these anuran species are: Ischnocnema manezinho (Garcia, 1996), Melanophryniscus spectabilis Caramaschi \& Cruz, 2002, Rhinella granulosa (Spix, 1824), Cycloramphus diringshofeni Bokermann, 1957, Proceratophrys appendiculata (Günther, 1873), Fritziana goeldii (Boulenger, 1895), Dendropsophus elianeae (Napoli \& Caramaschi, 2000), D. rubicundulus (Reinhardt \& Lütken, 1862), Boana geographica (Spix, 1824), B. guentheri (Boulenger, 1886), Phyllomedusa burmeisteri Boulenger, 1882, and Pithecopus hypochondrialis (Daudin, 1800). However, some of the taxa 


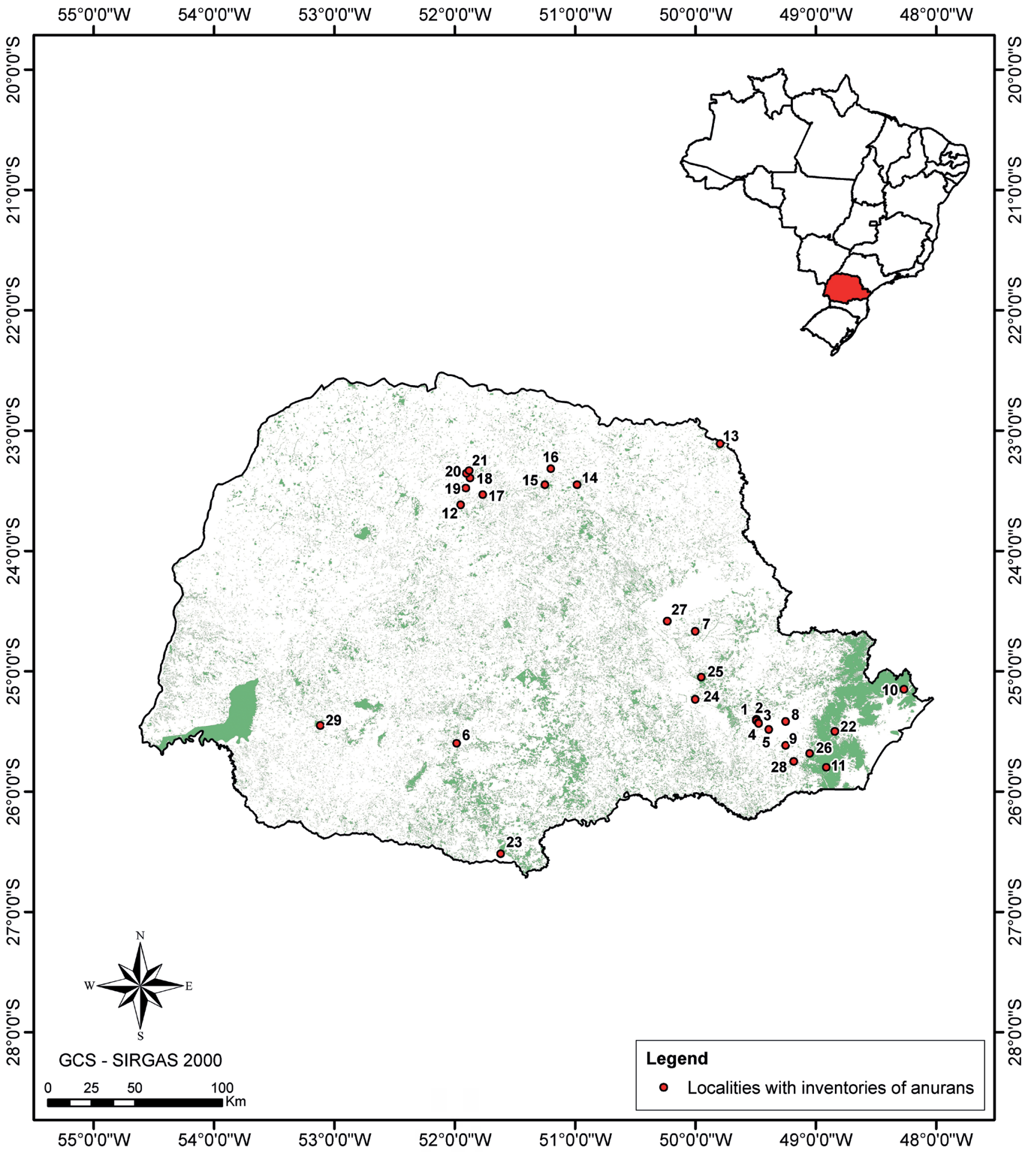

Figure 2. Municipalities with inventories of anurans in the state of Paraná, southern Brazil. 1-5 = Campo Largo (Leivas \& Hiert 2016); 6 = Candói, Pinhão and Foz do Jordão (Crivellari et al. 2014); 7 = Castro (Crivellari et al. 2014); $8=$ Curitiba (Crivellari et al. 2014); $9=$ Fazenda Rio Grande (Conte \& RossaFeres 2007); 10 = Guaraqueçaba (Garey \& Hartmann 2012, Santos-Pereira et al. 2011, 2016); 11 = Guaratuba (Cunha et al. 2010); 12 = Itambé (Affonso \& Delariva 2012); 13 = Jacarezinho (Nazaretti \& Conte 2015); 14-16 = Londrina (Affonso \& Delariva 2012, Benarde \& Anjos 1999, Machado et al. 1999); $17=$ Marialva (Affonso \& Delariva 2012); 18-21 = Maringá (Affonso et al. 2014); 22 = Morretes (Armstrong \& Conte 2010); 23 = Palmas (Crivellari et al. 2014); $24=$ Ponta Grossa (Crivellari et al. 2014); 25 = Ponta Grossa, Castro and Carambeí (Crivellari et al. 2014); 26 = São José dos Pinhais (Conte \& Rossa-Feres 2006); 27 = Tibagi (Crivellari et al. 2014); 28 = Tijucas do Sul (Conte \& Machado 2005); and 29 = Três Barras do Paraná (Bernarde \& Machado 2001). 
recorded in Paraná require revision. For example, eight species were identified "aff.", "cf." or "gr." in the articles consulted. One of them is Adenomera cf. bokermanni, cited by Santos-Pereira et al. (2016), and $A$. aff. bokermanni by Armstrong \& Conte (2010) and Garey \& Hartmann (2012). This species was originally described as Adenomera bokermanni by Heyer (1973), type locality in the municipality of Paranaguá, state of Paraná, and it seems very likely that the taxon cited in more recent studies corresponds to this species, given that these records refer to localities extremely close to the type locality of A. bokermanni, that is, municipalities of Morretes (Armstrong \& Conte 2010) and Guaraqueçaba (Garey \& Hartmann 2012, Santos-Pereira et al. 2016). Ischnocnema spanios (cited as "cf." in Santos-Pereira et al. 2016) is known to occur only from the minicipalyti of Boracéia (type locality of this species, Heyer, 1985) to the municipality of São Paulo (L. Malagoli, pers. comm.). Santos-Pereira et al. (2016) collected an anuran of the genus Cycloramphus, which they identified as $C$. cf. asper, although vouchers were juveniles (MNRJ 87917-87918), which hampered the identification of the species. The occurrence of Hylodes aff. asper by the first time by Garey \& Hartmann (2012) in the municipality of Guaraqueçaba may have been a mistake. Some years previously, Lingnau et al. (2008) described this species from a nearby municipality in the state of Paraná, Morretes, which is less than 90 $\mathrm{km}$ from Guaraqueçaba, and discussed the geographic distribution of Hylodes asper (Müller, 1924). For many years, herpetologists believed that the geographic range of $H$. asper extends from the state of Santa Catarina to the state of Rio de Janeiro, although this species has often been confused with $H$. perplicatus (Miranda-Ribeiro, 1926) or $H$. cardosoi Lingnau, Canedo \& Pombal, 2008. The geographic range of $H$. asper is now restricted to an area from the state of Rio de Janeiro to Paranapiacaba, municipality of Santo André in the state of São Paulo, while H. cardosoi, but not H. asper, is found in the south of state of $S \tilde{a} o$ Paulo (Lingnau et al. 2008). Therefore, we believe that the record of Hylodes aff. asper from Salto Morato, municipality of Guaraqueçaba, does in fact refers to $H$. cardosoi, but unfortunately, there are no voucher specimens or vocal records that might help confirm this hypothesis. Boana stellae (Kwet, 2008) is known only from the southern slope of the Araucaria Plateau in the center of the state of Rio Grande do Sul (Kwet 2008). Ololygon perpusillus is apparently restricted to the municipality of Rio de Janeiro (Peixoto 1987). Elachistocleis cesarii is known from northeastern Brazil, in the states of Ceará, Sergipe, and Bahia, central Brazil, in the states of Mato Grosso, Goiás, and Federal District, and southeastern Brazil, in the states of Minas Gerais, Espírito Santo, Rio de Janeiro and São Paulo (Caramaschi 2010). It is important to note that Fritziana gr. fissilis was recorded in two separate studies at the same locality, municipality of Guaraqueçaba, on the northern coast of the state of Paraná (Garey \& Hartmann 2012, Santos-Pereira et al. 2016). In this case, further fieldwork will be required to confirm the occurrence of the species in the state of Paranábecause the genus Fritziana requires a taxonomic review and the specimens cannot be assigned reliably to a specific species at the present time.

In addition to these taxonomically problematic anurans, several other species assigned to Paraná by Frost (2017) were not included in our list. As we found no other reliable evidence or records of the occurrence of these species in Paraná, we did not include them in our inventory. These species are Boana curupi (Garcia, Faivovich \& Haddad, 2007), Bokermannohyla claresignata (Lutz \& Lutz, 1939),
Trachycephalus imitatrix (Miranda-Ribeiro, 1926), Cycloramphus izecksohni Heyer, 1983 and Boana crepitans (Wied-Neuwied, 1824). In the case of $B$. claresignata, the only evidence is the record of Lutz (1973), who found tadpoles similar to those described for the species, but don't recorded any adult individual. Garcia et al. (2007) assumed that $B$. curupi occurs in Paraná, due to its association with the Paraná and Uruguay rivers, but we don't find any record of this anuran in the state of Paraná. Lutz (1973) found that the T. imitatrix specimens from southern Brazil (the "southern" form) were relatively large in size, and had a distinct type of iris, which is consistent with the description of T. dibernardoi Kwet \& Solé, 2008. The analysis of the specimens from the municipality of Telêmaco Borba revealed the larger body size and dorsal markings typical of $T$. dibernardoi (Conte et al. 2010). In addition, the description of $T$. dibernardoi for the region near Curitiba (Kwet \& Solé 2008) refers to a voucher specimen from a survey of the Fazenda Rio Grande (DZSJRP 8810; Conte \& Rossa-Feres 2007), where this anuran was confused with T. imitatrix (Conte et al. 2010). Given this evidence, we concluded that $T$. dibernardoi, rather than $T$. imitatrix, occurs in Paraná, as recorded by Lutz (1973), and that the occurrence of T. imitatrix recorded by Conte \& Rossa-Feres (2006, 2007) at São José dos Pinhais and Fazenda Rio Grande does in fact refer to $T$. dibernardoi. In the case of C. izecksohni, Frost (2017) only mentioned that the species occurs in the Serra do Mar coastal range, in the states of São Paulo, Paraná and Santa Catarina. Frost (2017) considered $B$. crepitans to occur in northern Brazil, with an allopatric population in the northeast, ranging as far south as Paraná and Santa Catarina. On these last two localities, we found available information about this anuran species only from the referred website.

Boana faber, Scinax fuscovarius, Dendropsophus minutus and Ischnocnema henselii were the anurans recorded most frequently in the studies analyzed. This reflects, in part, the wide geographic distribution of these species in Brazil (Frost 2017), as well as their life history strategies. In the case of the hylids, for example, populations are often associated with open areas or even disturbed habitats (Lucas \& Fortes 2008; Almeida-Gomes et al. 2010, 2014), resulting in a greater tolerance of these species to anthropogenic impacts in the environment. In particular, I. henseli, which is found in the leaf litter, was considered to be one of the dominant species of the anuran community at Guaraqueçaba (Santos-Pereira et al. 2011), given the considerable variety of microhabitats found in the leaf litter of the forest floor. This suggests that $I$. henseli besides frequent, is also an abundant leaf-litter frog found in the forest floor, and this possibly also indicates a greater environmental tolerance by this frog.

By contrast, 32 (23.3\%) of the 137 anuran species identified in the literature search were recorded in only one study, and most of these species are endemic to Paraná or occurs only also in the region that includes the neighboring states of Santa Catarina and São Paulo. While these records may reflect a relatively restricted distribution for these species, they may also be the result of subsampling in Paraná, given that research on anurans in this state is still incipient (Santos-Pereira 2016) with inventories of anuran species concentrated basically in two regions of state (see discussion below). The restricted occurrence of many species may also be related to the devastation of the state's forests, which has often impacted their structure, resulting in direct effects on their anuran species richness, including the possible eradication of the most sensitive forms. Only $11.7 \%$ of the original Atlantic Forest cover of 
Paraná now remains, and it has the highest historic rate of deforestation of any state of the Brazil (Fundação SOS Mata Atlântica \& Instituto Nacional de Pesquisas Espaciais 2015).

Eleven $(57.9 \%)$ of the 19 endemic species recorded in the present study are brachycephalids, and nine of these are Brachycephalus species, which is $26.5 \%$ of the 34 species currently recognized for this genus (Frost 2017). The species of this genus are found on the eastern coast of Brazil between the states of Bahia, in the northeast, and Santa Catarina, in the south (Frost 2017), with most occurring at altitudes of over $600 \mathrm{~m}$ in the Serra do Mar (Ribeiro et al. 2005, Alves et al. 2006). Many of these species have been described recently (Garey et al. 2012b, Ribeiro et al. 2015, 2017, Bornschein et al. 2016a), which emphasizes the importance of surveys in the least well-known remnants of Atlantic Forest, which are often found in relatively inaccessible areas of high altitude. The biodiversity corridors of the Atlantic Forest - the Central Atlantic Forest corridor, the Serra do Mar corridor, and the Northeast corridor (Rocha et al. 2003) - contain a considerable number of endemic vertebrates, including anuran amphibians, and the coast of Paraná is located within the largest continuous remnant of Atlantic Forest, which is part of the Serra do Mar corridor (Câmara 2005), which implies that the state may have more endemic anurans than currently known. This is consistent with the position of Pimm et al. (2010), who predicted that many endemic species of amphibians, birds, and mammals are yet to be discovered in the Brazil.

The type localities of the anuran species described from specimens collected in Paraná are concentrated in the east of the state. This probably reflects the greater research efforts in this region, where we recorded the highest number of localities with anuran inventories, and also to the principal remnants of native forest (Câmara 2005). Additionally, this can be reflected the flora inventories and the proximity of large urban centers or the presence of specialists in the taxonomic groups in these urban areas. Another factor is the presence (and density) of roads, which implies accessibility to natural areas (Pautasso 2007, Ficetola et al. 2013).

In the state of Paraná, the exotic invader anuran Lithobates catesbeianus has been recorded in 15 municipalities up until now (Santos-Pereira \& Rocha 2015). In general, these municipalities are located within a $60 \mathrm{~km}$ radius of commercial frog farms, indicating that, in most cases, the invasion of natural areas by L. catesbeianus has resulted from the escape of captive individuals into the natural environment (Santos-Pereira \& Rocha 2015). As the records show that L. catesbeianus is widely distributed in different regions of the state, it is possible that the actual area occupied by the bullfrog in the state of Paraná is much larger than currently thought. Further surveys in the state may provide new evidence on the extent of the area invaded by L. catesbeianus.

The red lists consulted during the present study revealed several inconsistencies in the conservation status of the anuran species identified in the state of Paraná. Some of these discrepancies may be the result of a certain asynchrony between the regional, national and global assessments (Rodríguez et al. 2000). The conservation status of nonendemic species will also vary according to the scale of analysis, even though it should be consistent among the state, national and global red lists (Brito et al. 2010). This indicates the existence of discrepancies between the national and global lists, given that two species endemic to the state of Paraná (B. pernix and I. paranaensis) are listed as DD by the IUCN, although I. paranaensis is not even listed by the Brazilian government. Similarly, Pseudis cardosoi is listed by the IUCN, but does not appear on the lists for Paraná or Brazil. In the specific case of the State list, the publication is more than 10 years old (Segalla \& Langone 2004), and requires revision, whereas on a broader scale, species not endangered in Paraná may be under threat in other states. As the regional lists are not updated regularly, they tend to provide outdated assessments of conservation status. This situation may reduce the effectiveness of these lists as guidelines for conservation practices, given that most measures are currently based on the evaluation of the extinction risk of the species (Miller et al. 2006). It is important to note that the regional lists are important conservation tools, given that most measures are implemented on a local scale (Possingham et al. 2002). Half of the anuran species recorded by us in this study that were categorized as data deficient in Paraná list or by the IUCN are endemic of this state. This reinforces the need for further research into the viability of their populations and the habitats they occupy, and their potential risk of extinction.

Based on the assessment of the IUCN (2017), most of the anuran species recorded in the present study - predominantly hylids - have stable populations. This is probably at least partly due to the fact that these are among the most abundant species in anuran communities, in particular in the Atlantic Forest (e.g., Conte \& Rossa-Feres 2006, Almeida-Gomes et al. 2010, Santos-Pereira et al. 2016). For example, the three species recorded most frequently in the publications analyzed in the present study (Boana faber, Dendropsophus anceps and Scinax fuscovarius) are known to be very common species (IUCN 2017), and $S$. fuscovarius is common even in deforested areas. The majority of leptodactylids have stable populations, and many of them [e.g., Adenomera marmorata Steindachner, 1867, Physalaemus cuvieri Fitzinger, 1826 and Leptodactylus mystaceus (Spix, 1824)], are common throughout their geographic ranges (IUCN 2017). Populations are declining in 26 species, which may reflect impacts on the ecosystems in which these anurans are found, in particular the high deforestation rates recorded throughout the state of Paraná. For example, Aplastodiscus ehrhardti (Müller, 1924), Proceratophrys brauni Kwet \& Faivovich, 2001 and Scythrophrys sawayae (Cochran, 1953) are relatively common species and their populations, while classified as declining by the IUCN, are stable in suitable habitats. Other example is Pleurodema bibroni, a rare species that occurs in widely scattered populations, being probably extinct in Montevideo Department, Uruguay, because of habitat destruction (IUCN 2017). The populations of only two anuran species listed here (Rhinella schneideri and Lithobates catesbeianus) are thought to be increasing (IUCN 2017). Lithobates catesbeianus is widespread in Paraná (Santos-Pereira \& Rocha 2015). This species is known worldwide from thousands of localities and is usually very abundant with increasing populations (IUCN 2017). In 28 cases, mostly brachycephalids and bufonids, population trends are unclear, although some brachycephalids, such as Brachycephalus brunneus, B. ferruginus, B. izecksohni, Ischnocnema henselii and I. sambaqui, are locally abundant (IUCN 2017). No data are available on the population trends of other brachycephalids, i.e., B. pombali and I. spanios (IUCN 2017). The populations of the Brachycephalus species are typically restricted to fragments of hill forests (Pombal et al. 1998, Ribeiro et al. 2005, Alves et al. 2006), separated by valleys, which isolates the populations (Pie et al. 2013) and makes them difficult to survey effectively. In the case 
of the bufonids, Dendrophryniscus stawiarskyi, for example, is known only from three specimens collected in the early 1980s, and there is currently no information on its population status, while the species of the genus Melanophryniscus, M. alipioi and M. tumifrons (Boulenger, 1905), are extremely difficult to survey, although the former species is commonly encountered during the breeding season (IUCN 2017). This possibly is due to the fact that the anuran species of this genus show explosive breeding, reproducing in small and ephemeral water bodies (e.g. Cairo et al. 2008, Laufer et al. 2015). Given this scenario, we identified three main variables that are important for the evaluation of the population viability of anurans - the abundance of the species, the difficulty of capturing specimens and the quality of the environment inhabited by the population.

While we identified an additional 10 anuran species during the survey of the scientific collections available in the SpeciesLink database, we did not include these species in our inventory of the anurans of the state of Paraná. The record of Allobates brunneus, for example, is almost certainly erroneous, given that the genus Allobates is not found in Paraná (Verdade \& Rodrigues 2007). While it may be possible that Vitreorana eurygnatha occurs in Paraná, we found only a single specimen in the Brazilian National Museum of Natural History (NMNH), so at this time, we prefer to exercise caution, and not confirm that the species occurs in Paraná. Similarly, we have classified Aplastodiscus cochranae, A. leucopygius, Crossodactylus gaudichaudii, Leptodactylus chaquensis and Physalaemus biligonigerus as tentative occurrences, pending the confirmation of the taxonomic identification of the specimens. In the specific case of Boana polytaenia, we consider the identification doubtful, given that Cruz \& Caramaschi (1998) did not refer to the occurrence of the species in Paraná in their review [the only species of this complex known unequivocally to occur in the state of Paraná, at the present time, and that was included in our list, is Boana jaguariavensis (Caramaschi, Cruz \& Segalla, 2010)]. It seems likely the record of Ololygon brieni does in fact refers to O. littoralis (Pombal \& Gordo 1991). Finally, it does seem likely that Pseudopaludicola falcipes occurs in Paraná, given that this species is cited by Frost (2017).

The inventories of anurans in the state of Paraná have been concentrated primarily in the metropolitan region of the Curitiba city, in the eastern extreme, and the central-northern region of the state, while there are severalgeographic distribution gaps in most of the state. This concentration of research in the north and east of the state reflects the proximity of its principal universities, including the Federal University of Paraná, in the Curitiba city, and the State universities of Londrina and Maringá, both located in the central-northern region. The metropolitan region of Curitiba, which extends as far as the Atlantic coast, also encompasses several other institutions, including universities and museums, and a major portion of the largest continuous remnant of Atlantic Rainforest found in Brazil. This region also contains a number of protected areas, which may stimulate the interest of researchers seeking well-preserved environments and faunal communities. It is interesting to note that there has been no inventory of anurans in the Foz do Iguaçu micro-region, in western Paraná, which includes the Iguaçu National Park, one of Brazil's first national parks, created in 1939.

This study was the first attempt to compile a comprehensive inventory of the anuran fauna of the Brazilian state of Paraná. We consider our list of species to be a relatively reliable estimate of the anuran diversity of the state, although we do expect new species to be added to the list, in particular because many areas have yet to be sampled adequately. In addition to the need for more extensive surveys, especially in areas that have yet to be sampled adequately, a number of forms require taxonomic review. We also emphasize the need for the conservation of the state's remaining natural habitats, as well as further research on the invasion of the region by the exotic frog, Lithobates catesbeianus, especially in relation to the escape and dispersal of animals from local frog farms.

\section{Acknowledgments}

This study was supported by research grants from the Conselho Nacional de Desenvolvimento Científico e Tecnológico (CNPq) to CFD Rocha (processes 302974/2015-6 and 424473/2016-0) and to JP Pombal Jr. (processes 440501/2015-6, 474911/2013-6, 305382/2013-6) and from Fundação de Amparo à Pesquisa do Estado do Rio de Janeiro (FAPERJ) through "Cientistas do Nosso Estado" Program to CFD Rocha (process E-26/202.920.2015) and to JP Pombal Jr. (process E-26/203.014/2016). M. Santos-Pereira receives Post Doc fellowship from CNPq. We also thank L. Malagoli for information on the geografic distribution of $I$. spanios.

\section{Author Contributions}

Manuela Santos-Pereira: Substantial contribution to the conception and design of the work; Contribution in the acquisition of the data; Contribution in the analysis and interpretation of the data; Contribution in the writing of the work; Contribution in the critical review appending intellectual content.

José P. Pombal Jr.: Substantial contribution in the acquisition of the data; contribution in the analysis and interpretation of the data; contribution in the writing of the work; contribution in the critical review appending intellectual content.

Carlos Frederico Duarte Rocha: Substantial contribution to the conception and design of the work; contribution in the acquisition of the data; contribution in the analysis and interpretation of the data; contribution in the writing of the work; contribution in the critical review appending intellectual content.

\section{Conflicts of interest}

The authors declare that they have no conflict of interest related to the publication of this manuscript.

\section{References}

AFFONSO, I.P., DELARIVA, R.L. \& NAVARRO, M.P. 2011. Amphibia, Anura, Leptodactylidae, Leptodactylus mystaceus (Spix, 1824): Distribution extension. Check List 7(2): 198-199.

AFFONSO, I.P. \& DELARIVA, R.L. 2012. Lista comentada da anurofauna de três municípios da região Noroeste do Estado do Paraná, Brasil. SaBios: Revista Saúde e Biologia 7(2): 102-109.

AFFONSO, I.P., CAFOFO, E.G., DELARIVA, R.L., ODA, F.H., KARLING, L.C. \& LOURENÇO-DE-MORAES, R. 2014. List of anurans (Amphibia: Anura) from the rural zone of the municipality of Maringá, Paraná state, southern Brazil. Check List 10(4): 878-882. doi: 10.15560/10.4.878

ALMEIDA, A.P., GASPARINI, J.L. \& PELOSO, P.L.V. 2011. Frogs of the state of Espírito Santo, Southeastern Brazil - The need for looking at the 'coldspots'. Check List 7(4): 542-560. 
ALMEIDA, J.P.F.A., NASCIMENTO, F.A.C., TORQUATO, S., LISBOA, B.S., TIBURCIO, I.C.S., PALMEIRA, C.N.S., LIMA, M.G. \& MOTT, T. 2016. Amphibians of Alagoas State, northeastern Brazil. Herpetology Notes 9: 123-140

ALMEIDA-GOMES, M., ALMEIDA-SANTOS, M., GOYANNES-ARAÚJO, P., BORGES-JR., V.N.T., VRCIBRADIC, D., SIQUEIRA, C.C., ARIANI, C.V., DIAS, A.S., SOUZA, V.V., PINTO, R.R., VAN SLUYS, M. \& ROCHA, C.F.D. 2010. Anurofauna of an Atlantic Rainforest fragment and its surroundings in northern Rio de Janeiro State, Brazil. Brazilian Journal of Biology 70(3): 871-877. doi: 10.1590/S1519- 69842010000400018

ALMEIDA-GOMES, M., SIQUEIRA, C.C., BORGES-JR., V.N.T., VRCIBRADIC, D., FUSINATTO, L.A. \& ROCHA, C.F.D. 2014. Herpetofauna of the Reserva Ecológica de Guapiaçu (REGUA) and its surrounding areas, in the state of Rio de Janeiro, Brazil. Biota Neotropica 14(3): 1-15. http://dx.doi.org/10.1590/1676-0603007813 (last access on 06/11/2017).

ALVES, A.C.R., RIBEIRO, L.F., HADDAD, C.F.B. \& REIS, S.F. 2006. Two new species of Brachycephalus (Anura: Brachycephalidae) from the Atlantic Forest in Paraná State, Southern Brazil. Herpetologica 62(2): 221-233.

ANTONUCCI, A.M., ODA, F.H., SIGNORELLI, L., SANTANA, N.F. \& MENDES, M.C. 2011. Parasitismo de Amblyomma rotundatum (Koch, 1844) (Acari: Ixodidae) em Rhinella schneideri (Werner, 1894) (Anura: Bufonidae) no estado do Paraná, Brasil. Natureza on line 9(3): 103-105.

ARAÚJO, O.G.S., TOLEDO, L.F., GARCIA, P.C.A. \& HADDAD, C.F.B. 2009. The Amphibians of São Paulo State, Brazil. Amphibians of São Paulo. Biota Neotropica 9(4): 197-209. http://www.biotaneotropica.org.br/v9n4/ pt/abstract?article+bn03109042009 (last access on 11/06/2017).

ARMSTRONG, C.G. \& CONTE, C.E. 2010. Taxocenose de anuros (Amphibia: Anura) em uma área de Floresta Ombrófila Densa no Sul do Brasil. Biota Neotropica 10(1): 39-46. http://www.biotaneotropica.org.br/v10n1/pt/ abstract?article+bn00610012010 (last access on 11/06/2017).

BALDO, D., TOMATIS, C. \& SEGALlA, M.V. 2008. Amphibia, Anura, Leptodactylidae, Leptodactylus furnarius: New country record, geographic distribution map and advertisement call. Check List 4(2): 98-102.

BERNARDE, P.S. \& ANJOS, L. 1999. Distribuição especial e temporal da anurofauna no Parque Estadual Mata dos Godoy, Londrina, Paraná, Brasil (Amphibia: Anura). Comunicações do Museu de Ciências e Tecnologia da PUCRS. Série Zoologia 12: 111-140.

BERNARDE, P.S. \& MACHADO, R.A. 2001. Riqueza de espécies, ambientes de reprodução e temporada de vocalização da anurofauna em Três Barras do Paraná, Brasil (Amphibia: Anura). Cuadernos de Herpetología 14(2): 93-104.

BERNECK, B.V., HADDAD, C.F., LYRA, M.L., CRUZ, C.A. \& FAIVOVICH, J. 2016. The Green Clade Grows: a phylogenetic analysis of Aplastodiscus (Anura; Hylidae). Molecular Phylogenetics and Evolution 97: 213-223.

BOKERMANN, W.C.A. 1965. Hyla langei, a new frog from Paraná, southern Brasil. Journal of the Ohio Herpetological Society 5(2): 49-51.

BORNSCHEIN, M. R., BELMONTE-LOPES, R., RIBEIRO, L. F., MAURICIO, G. N. \& PIE, M. R. 2015a. Rectification of the position of the type locality of Brachycephalus tridactylus (Anura: Brachycephalidae), a recently described species from southern Brazil. Zootaxa 4007(1): 149-150.

BORNSCHEIN, M.R., MAURÍCIO, G.N. \& PIE, M.R. 2015b. Rectification of the type locality of Ischnocnema paranaensis (Anura: Brachycephalidae), a missing species of the Atlantic Forest of Brazil. Zootaxa 3957: 249-250.

BORNSCHEIN, M.R., RIBEIRO, L.F., BLACKBURN, D.C., STANLEY, E.L. \& PIE, M.R. 2016a. A new species of Brachycephalus (Anura: Brachycephalidae) from Santa Catarina, southern Brazil. PeerJ 4:e2629. https://doi.org/10.7717/peerj.2629 (last access on 11/06/2017).

BORNSCHEIN, M.R., FIRKOWSKI, C.R., BELMONTE-LOPES, R., CORRÊA, L., RIBEIRO, L.F., MORATO, S.A., ANTONIAZZI-JR. R.L., REINERT, B.L., MEYER, L.S., CINI, A. \& PIE, M.R. 2016b. Geographical and altitudinal distribution of Brachycephalus (Anura: Brachycephalidae) endemic to the Brazilian Atlantic Rainforest. PeerJ 4, e2490. https://doi. org/10.7717/peerj.2490 (last access on 11/06/2017).
BRITO, D., AMBAL, R.G., BROOKS, T., DE SILVA, N., FOSTER, M., HAO, W., HILTON-TAYLOR, C., PAGLIA, A.P., RODRIGUEZ, J.P. \& RODRIGUEZ, J.V. 2010. How similar are National Red Lists and the IUCN Red List? Biological Conservation 143: 1154-1158.

CAIRO, S.L., ZALBA, S.M., \& ÚBEDA, C.A. 2008. Reproductive behaviour of Melanophryniscus sp. from Sierra de la Ventana (Buenos Aires, Argentina). South American Journal of herpetology 3(1): 10-14.

CÂMARA, I.G. 2005. Breve história da conservação da Mata Atlântica. In Mata Atlântica: Biodiversidade, ameaças e perspectivas. (C. Galindo-Leal, I.G. Câmara, eds). Conservação Internacional, Belo Horizonte, p.31-42.

CARAMASCHI, U. \& CRUZ, C.A.G. 2002. Taxonomic status of Atelopus pachyrhynus Miranda-Ribeiro, 1920, redescription of Melanophryniscus tumifrons (Boulenger, 1905), and descriptions of two new species of Melanophryniscus from the State of Santa Catarina, Brazil (Amphibia, Anura, Bufonidae). Arquivos do Museu Nacional 60(4): 303-314.

CARAMASCHI, U. \& RODRIGUES, M.T. 2007. Taxonomic status of the species of Gastrotheca Fitzinger, 1843 (Amphibia, Anura, Amphignathodontidae) of the Atlantic Rain Forest of eastern Brazil, with description of a new species. Boletim do Museu Nacional 525: 1-19.

CARAMASCHI, U., CRUZ, C.A.G. \& SEGALLA, M.V. 2010. A new species of Hypsiboas of the H. polytaenius clade from the State of Paraná, Southern Brazil (Anura: Hylidae). South American Journal of Herpetology 5: 169-174.

CARAMASCHI, U. 2010. Notes on the taxonomic status of Elachistocleis ovalis (Schneider, 1799) and description of five new species of Elachistocleis Parker, 1927 (Amphibia, Anura, Microhylidae). Boletim do Museu Nacional. Nova Serie, Zoologia 527: 1-30.

CASTANHO, L.M. \& HADDAD, C.F.B. 2000. New species of Eleutherodactylus (Amphibia: Leptodactylidae) from Guaraqueçaba, Atlantic Forest of Brazil Copeia 3: 777-781.

CLEMENTE-CARVALHO, R.B.G., ANTONIAZZI, M.M., JARED, C., HADDAD, C.F.B., ALVES, A.C.R., ROCHA, H.S., PEREIRA, G.R., OLIVEIRA, D.F., LOPES, R.T. \& REIS, S.F. 2009. Hyperossification in niniaturized toadlets of the genus Brachycephalus (Amphibia: Anura: Brachycephalidae): Microscopic structure and macroscopic oatterns of variation. Journal of Morphology 270: 1285-1295.

CLEMENTE-CARVALHO, R.B.G., KLACZKO, J., ALVES, A.C.R., HADDAD, C.F.B. \& REIS, S.F. 2011. Molecular phylogenetic relationships and phenotypic diversity in miniaturized toadlets, genus Brachycephalus (Amphibia: Anura: Brachycephalidae). Molecular Phylogenetics and Evolution 61: 79-89.

CONDEZ, T.H., MONTEIRO, J.P.C., COMITTI, E.J., GARCIA, P.C.A., AMARAL, I.V. \& HADDAD, C.F.B. 2016. A new species of flea-toad (Anura: Brachycephalidae) from southern Atlantic. Zootaxa 4083(1): 40-56.

CONTE, C.E., LINGNAU, R. \& KWET, A. 2005. Description of the advertisement call of Hyla ehrhardti MÜLLER, 1924 and new distribution records (Anura: Hylidae). Salamandra 41(3): 147-151.

CONTE, C.E. \& MACHADO, R.A. 2005. Riqueza de espécies e distribuição espacial e temporal em comunidade de anuros (Amphibia, Anura) em uma localidade de Tijucas do Sul, Paraná, Brasil. Revista Brasileira de Zoologia 22(4): 940-948.

CONTE, C.E., NOMURA, F., MACHADO, R.A., KWET, A., LINGNAU, R. \& ROSSA-FERES, D.C. 2010. Novos registros na distribuição geográfica de anuros na Floresta com Araucária e considerações sobre suas vocalizações. Biota Neotropica 10(2): 201-224.

CONTE, C.E. \& ROSSA-FERES, D.C. 2006. Diversidade e ocorrência temporal da anurofauna (Amphibia, Anura) em São José dos Pinhais, Paraná, Brasil. Revista Brasileira de Zoologia 23(1): 162-175.

CONTE, C.E. \& ROSSA-FERES, D.C. 2007. Riqueza e distribuição espaçotemporal de anuros em um remanescente de Floresta de Araucária no sudeste do Paraná. Revista Brasileira de Zoologia 24(4): 1025-1037.

COSTA, T.R.N., LINGNAU, R. \& TOLEDO, L.F. 2009. The tadpole of the Brazilian torrent frog Hylodes heyeri (Anura; Hylodidae). Zootaxa 2222: 66-68. 
CRIVELLARI, L.B., LEIVAS, P.T., MOURA LEITE, J.C., GONÇALVES, D.S., MELLO, C.M., ROSSA-FERES, D.C. \& CONTE, C.E. 2014. Amphibians of grasslands in the state of Paraná, southern Brazil (Campos Sulinos). Herpetology Notes 7: 639-654.

CRUZ, C.A.G. 1990. Sobre as relações intergenéricas de Phyllomedusinae da Floresta Atlântica (Amphibia, Anura, Hylidae). Revista Brasileira de Biologia 50(3): 709-726.

CRUZ, C.A.G. \& CARAMASCHI, U. 1998. Definição, composição e distribuição geográfica do grupo de Hyla polytaenia Cope, 1870 (Amphibia, Anura, Hylidae). Boletim do Museu Nacional (N.S.) Zoologia (392): 1-19.

CRUZ, C.A.G., CASSINI, C.S. \& CARAMASCHI, U. 2008. A New Species of the Genus Physalaemus Fitzinger, 1826 (Anura, Leiuperidae) from Southern Brazil. South American Journal of Herpetology 3(3): 239-243.

CUNHA, A.K., OLIVEIRA, I.S. \& HARTMANN, M.T. 2010. Anurofauna da Colônia Castelhanos, na Área de Proteção Ambiental de Guaratuba, Serra do Mar paranaense, Brasil. Biotemas 23(2): 123-134.

FICETOLA, G.F., BONARDI, A., SINDACO, R., PADOA-SCHIOPPA, E 2013. Estimating patterns of reptile biodiversity in remote regions. Journal of Biogeography 40: 1202-1211.

FIGUEIREDO, G.T., SANTANA, D.J. \& DOS ANJOS, L. 2014. New records and distribution map of Scinax rizibilis (Bokermann, 1964). Herpetology Notes 7: 531-534.

FONTOURA, P.L., RIBEIRO, L.F. \& PIE, M.R. 2011. Diet of Brachycephalus brunneus (Anura: Brachycephalidae) in the Atlantic Rainforest of Paraná, southern Brazil. Zoologia 28(5): 687-689.

FROST, D.R. 2017. Amphibian Species of the World: an Online Reference. Version 6.0. American Museum of Natural History, New York, USA. Available online at: http://research.amnh.org/vz/herpetology/amphibia/ index.php. (last access on 09/12/2017).

FUNDAÇÃO SOS MATA ATLÂNTICA \& INSTITUTO NACIONAL DE PESQUISAS ESPACIAIS (INPE). 2015. Atlas dos Remanescentes Florestais da Mata Atlântica Período 2013-2014. Relatório Técnico. São Paulo, 60p. http://mapas.sosma.org.br/site_media/download atlas_2013-2014_relatorio_tecnico_2015.pdf (last access on 15/11/2016).

GAMBALE, P.G., BATISTA, V.G., ODA, F.H., CAMPOS, R.M., TAKEMOTO, R.M. \& BASTOS, R.P. 2014. Anuran larvae as prey and hosts of invertebrates in Neotropical aquatic habitats. Revista Chilena de Historia Natural 87(31): 1-5.

GARCIA, P.C.A., FAIVOVICH J., \& HADDAD, C.F.B. 2007. Redescription of Hypsiboas semiguttatus, with the description of a new species of the Hypsiboas pulchellus group. Copeia 2007(4): 933-951.

GAREY, M.V., COSTA, T.R.N., LIMA, A.M.X., TOLEDO, L.F. \& HARTMANN, M.T. 2012a. Advertisement call of Scinax littoralis and S. angrensis (Amphibia: Anura: Hylidae), with notes on the reproductive activity of S. littoralis. Acta Herpetologica 7(2): 297-308.

GAREY, M.V., LIMA, A.M.X., HARTMANN, M.T. \& HADDAD, C.F.B. 2012b. A new species of miniaturized toadlet, genus Brachycephalus (Anura: Brachycephalidae), from Southern Brazil. Herpetologica 68(2): 266-271.

GAREY, M.V. \& HARTMANN, M.T. 2012. Anuros da Reserva Natural Salto Morato, Guaraqueçaba, Paraná, Brasil. Biota Neotropica 12(4): 137-145.

HEYER, W.R. 1973. Systematics of the marmoratus group of the frog Genus Leptodactylus (Amphibia, Leptodactylidae). Natural History Museum of Los Angeles County. Science Bulletin 251: 1-27.

HEYER, W.R. 1978. Systematics of the fuscus group of the Frog Genus Leptodactylus (Amphibia, Leptodactylidae). Natural History Museum of Los Angeles County. Science Bulletin 29:1-89.

HEYER, W.R. 1983. Variation and systematics of frogs of the genus Cycloramphus (Amphibia, Leptodactylidae). Arquivos de Zoologia 30(4): 235-339.

HEYER, W.R. 1985. New species of frogs from Boracéia, São Paulo, Brazil Procceding of Biological Society of Washington 98: 657-671.

HEYER, W.R. \& HEYER, M.M. 2004. "Leptodactylus furnarius". Catalogue of American Amphibians and Reptiles 785: 1-5.

HIERT, C.E. \& MOURA, M.O. 2010. Abiotic correlates of temporal variation of Hypsiboas leptolineatus (Amphibia: Hylidae). Zoologia 27(5): 703-708
HIERT, C.E., ROPER, J.J. \& MOURA, M.O. 2012. Constant breeding and low survival rates in the subtropical striped frog in southern Brazil. Journal of Zoology 288(2): 151-158.

IUCN (INTERNATIONAL UNION FOR CONSERVATION OF NATURE). 2017. IUCN Red List of threatened species. Version 2011.2. http://www. iucnredlist.org/ (last access on 08/11/2017).

IZECKSOHN, E. 1993. Três novas espécies de Dendrophryniscus Jiménez de La Espada das regiões sudeste e sul do Brasil (Amphibia, Anura, Bufonidae) Revista Brasileira de Zoologia 10(3): 473-488.

KWET, A. 2008. New species of Hypsiboas (Anura: Hylidae) in the pulchelus group from southern Brazil. Salamandra 44: 1-14.

KWET, A. \& SOLÉ, M. 2008. A new species of Trachycephalus (Anura: Hylidae) from the Atlantic Rain Forest in southern Brazil. Zootaxa 1947: 53-67.

LANGONE, J.A. \& SEGALLA, M.V. 1996. Una nueva especie de Eleutherodactylus del Estado de Paraná, Brasil. Comunicaciones Zoológicas del Museo de Historia Natural de Montevideo 12: 1-5.

LANGONE, J.A., SEGALLA, M.V., BORNSCHEIN, M. \& DE SÁ, R.O. 2008 A new reproductive mode in the genus Melanophryniscus Gallardo, 1961 (Anura: Bufonidae) with description of a new species from the state of Paraná, Brazil. South American Journal of Herpetology 3: 1-9.

LAUFER, G., VAIRA, M., PEREYRA, L.C., \& AKMENTINS, M.S. 2015. The use of ephemeral reproductive sites by the explosive breeding toad Melanophryniscus rubriventris (Anura: Bufonidae): is it a predator cue mediated behavior? Studies on Neotropical Fauna and Environment 50(3): 175-181.

LEIVAS, P.T. \& HIERT, C. 2016. Anuran richness in remnants of Araucaria Forest, Paraná, Brazil. Herpetology Notes 9: 15-21.

LIMA, A.M.X., GAREY, M.V., NOLETO, R.B. \& VERDADE, V.K. 2010. Natural History of the Lutz's Frog Cycloramphus lutzorum Heyer, 1983 (Anura: Cycloramphidae) in the Brazilian Atlantic Forest: Description of the advertisement call, tadpole, and karyotype. Journal of Herpetology 44(3): 360-371.

LINGNAU, R. \& BASTOS, R.P. 2007. Vocalizations of the Brazilian torrent frog Hylodes heyeri (Anura: Hylodidae): Repertoire and influence of air temperature on advertisement call variation. Journal of Natural History 41: 1227-1235

LINGNAU, R., CANEDO, C. \& POMBAL JR., J.P. 2008. A new species of Hylodes (Anura: Hylodidae) from the Brazilian Atlantic Forest. Copeia 3: 595-602.

LUCAS, E.M. \& FORTES, V.B. 2008. Frog diversity in the Floresta Nacional de Chapecó, Atlantic Forest of southern Brazil. Biota Neotropica 8(3): 51-61.

LUTZ, B. 1973. Brazilian Species of Hyla. University of Texas Press, Austin.

MACHADO, I.B. \& MALTCHIK, L. 2007. Check-list da diversidade de anuros no Rio Grande do Sul (Brasil) e proposta de classificação para as formas larvais. Neotropical Biology and Conservation 2(2): 101-116.

MACHADO, R.A., BERNARDE, P.S., MORATO, S.A.A. \& DOS ANJOS, R. 1999. Análise comparada da riqueza de anuros entre duas áreas com diferentes estados de conservação no município de Londrina, Paraná, Brasil (Amphibia, Anura). Revista Brasileira de Zoologia 16(4): 997-1004.

MACHADO, R.A. \& BERNARDE, P.S. 2002. Anurofauna da bacia do Rio Tibagi. In A bacia do Rio Tibagi (M.E. Medri, E. Bianchini, O.A. Shibatta \& J.A. Pimenta eds.). Medri, Londrina, pp. 297-306.

MARCELINO, V.R., HADDAD, C.F.B. \& ALEXANDRINO, J. 2009. Geographic distribution and morphological variation of striped and nonstriped populations of the Brazilian Atlantic Forest treefrog Hypsiboas bischoffi (Anura: Hylidae). Journal of Herpetology 43(2): 351-361.

MILLER, R.M., RODRÍGUEZ, J.P., ANISKIEWICZ-FOWLER, T., BAMBARADENIYA, C., BOLES, R., EATON, M.A., GARDENFORS, U., KELLER, V., MOLUR, S., WALKER, S. \& POLLOCK, C. 2006. Extinction risk and conservation priorities. Science 313: 441

MIRANDA, D.B., GAREY, M.V., MONTEIRO-FILHO, E.L.A. \& HARTMANN, M.T. 2008. Sinalização visual e biologia reprodutiva de Dendropsophus werneri (Anura: Hylidae) em área de Mata Atlântica no Estado do Paraná, Brasil. Papéis Avulsos de Zoologia 48(29): 335-343. 
MMA. 2014. Portaria $N^{\circ} 444$, de 17 de Dezembro de 2014. Lista Nacional Oficial de Espécies da Fauna Ameaçadas de Extinção.

MORESCO, R.M., MARGARIDO, V.P., NAZARIO, P.D., SCHMIT, R.A. \& TRECO, F.R. 2009. Amphibia, Anura, Hylidae, Scinax granulatus: Distribution extension. Check List 5(1): 086-088.

MORESCO, R.M., MANIGLIA, T.C., DE OLIVEIRA, C. \& MARGARIDO, V.P. 2013. The pioneering use of ISSR (Inter Simple Sequence Repeat) in Neotropical anurans: preliminary assessment of genetic diversity in populations of Physalaemus cuvieri (Amphibia, Leiuperidae). Biological Research 46: 53-57.

MORESCO, R.M., DE OLIVEIRA, C. \& MARGARIDO, V.P. 2014. A persistent organic pollutant related with unusual high frequency of hermaphroditism in the neotropical anuran Physalaemus cuvieri Fitzinger, 1826. Environmental Research 132: 6-11.

MYERS, N., MITTERMEIER, R., MITTERMEIER, C., FONSECA, G. \& KENT, J. 2000. Biodiversity hotspots for conservation priorities. Nature 403: $853-858$.

NAZARETTI, E.M. \& CONTE, C.E. 2015. Anurofauna de um remanescente alterado de floresta estacional semidecidual as margens do Rio Paranapanema. Iheringia, Série Zoologia 105(4): 420-429.

NUNES, I., KWET, A. \& POMBAL JR., J.P. 2012. Taxonomic revision of the Scinax alter Species complex (Anura: Hylidae). Copeia 3: 554-569.

ODA, F.H. \& LANDGRAF, G.O. 2012. An unusual case of scavenging behavior in Rhinella schneideri in the upper Paraná River basin, Brazil. Boletín de la Asociación Herpetológica Española 23(1): 57-59.

ODA, F.H., PETSCH, D.K., RAGONHA, F.H., BATISTA, V.G., TAKEDA, A.M. \& TAKEMOTO, R.M. 2015. Dero (Allodero) lutzi Michaelsen, 1926 (Oligochaeta: Naididae) associated with Scinax fuscovarius (Lutz, 1925) (Anura: Hylidae) from Semi-deciduous Atlantic Rain Forest, southern Brazil. Brazilian Jounal of Biology 75(1): 86-90.

OLIVEIRA, I.S., OLIVEIRA, A.K.C., CESTARI, M.M. \& TOLEDO, L.F. 2010. Predation on Dendropsophus werneri (Anura: Hylidae) by a lycosid in the Atlantic forest, southern Brazil. Herpetology Notes 3: 299-300.

PAUTASSO, M. 2007. Scale dependence of the correlation between human population presence and vertebrate and plant species richness. Ecology Letters 10: 16-24.

PEDERASSI, J., LIMA, M.S.C.S. \& SOUZA, C.A.S. 2015. Scinax nasicus (Lesser Snouted Treefrog). Herpetological Review 46(2): 214.

PEIXOTO, O.L. 1987. Caracterização do grupo "perpusilla" e reavaliação da posição taxonômica de Ololygon perpusilla perpusilla e Ololygon perpusilla $v$-signata (Amphibia, Anura, Hylidae). Arquivos da Universidade Federal do Rural do Rio de Janeiro 10: 37-49.

PIE, M.R., MEYER, A.L.S., FIRKOWSKI, C.R., RIBEIRO, L.F. \& BORNSCHEIN, M.R. 2013. Understanding the mechanisms underlying the distribution of microendemic montane frogs (Brachycephalus spp., Terrarana: Brachycephalidae) in the Brazilian Atlantic Rainforest. Ecological Modelling 250: 165-176.

PIMENTA, B.V.S., CRUZ, C.A.G. \& CARAMASCHI, U. 2014. Taxonomic Review of the Species Complex of Crossodactylus dispar A. lutz, 1925 (Anura, Hylodidae). Arquivos de Zoologia 45: 1-33.

PIMM, S.L., JENKINS, C.N., JOPPA, L.N., ROBERTS, D.L. \& RUSSELL, G.J. 2010. How many endangered species remain to be discovered in Brazil? Natureza \& Conservação 8: 71-77.

POMBAL JR., J.P., WISTUBA, E. \& BORNSCHEIN, M. 1998. A new species of brachycephalid (Anura) from the Atlantic rainforest of Brazil. Journal of Herpetology 32(1): 70-74.

POSSINGHAM, H.P., ANDELMAN, S.J., BURGMAN, M.A., MEDELLIN, R.A., MASTER, L.L. \& KEITH, D.A. 2002. Limits to the use of threatened species lists. Trends in Ecology \& Evolution 17(11): 503-507.

PRADO, G.M. \& POMBAL JR., J.P. 2008. Espécies de Proceratophrys MirandaRibeiro, 1920 com apêndices palpebrais (Anura; Cycloramphidae). Arquivos de Zoologia 39(1): 1-85.
RIBEIRO, L.F., ALVES, A.C.R. \& HADDAD, C.F.B. 2005. Two new species of Brachycephalus Günther, 1858 from the state of Paraná, southern Brazil (Amphibia, Anura, Brachycephalidae). Boletim do Museu Nacional (Zoologia) 519: 1-18.

RIBEIRO, L.F., BORNSCHEIN, M.R., BELMONTE-LOPES, R., FIRKOWSKI, C.R., MORATO, S.A.A. \& PIE, M.R. 2015. Seven new microendemic species of Brachycephalus (Anura: Brachycephalidae) from southern Brazil. PeerJ 3:e1011. 1-35. https://doi.org/10.7717/peerj.1011(last access on 11/06/2017).

RIBEIRO, L.F., BLACKBURN, D.C., STANLEY, E.L., PIE, M.R. \& BORNSCHEIN, M.R. 2017. Two new species of the Brachycephalus pernix group (Anura: Brachycephalidae) from the state of Paraná, southern Brazil. PeerJ 5, e3603. https://doi.org/10.7717/peerj.3603 (last access on 11/06/2017).

ROBERTO, I.J., RIBEIRO, S.C. \& LOEBMANN, D. 2013. Amphibians of the state of Piauí, Northeastern Brazil: a preliminary assessment. Biota Neotropica 13(1): 322-330. http://www.biotaneotropica.org.br/v13n1/en/ abstract?article+bn04113012013 (last access on 11/06/2017).

ROCHA, C.F.D., BERGALLO, H.G., ALVES, M.A.S. \& VAN SLUYS, M. 2003. A biodiversidade nos grandes remanescentes florestais do Estado do Rio de Janeiro e nas Restingas da Mata Atlântica. Rima Editora, São Carlos.

ROCHA, C.F.D., BERGALLO, H.G., POMBAL JR., J.P., GEISE, L., VAN SLUYS, M., FERNANDES, R. \& CARAMASCHI, U. 2004. Fauna de anfíbios, répteis e mamíferos do Estado do Rio de Janeiro, Sudeste do Brasil. Publicações Avulsas do Museu Nacional 104: 1-24.

RODRÍGUEZ, J.P., ASHENFELTER, G., ROJAS-SUÁREZ, F., GARCIA FERNANDEZ, J.J., SUÁREZ, L. \& DOBSON, A.P. 2000. Local data are vital to worldwide conservation. Nature 403: 241.

ROSSA-FERES, D.C., SAWAYA, R.J., FAIVOVICH, J., GIOVANELLI, J.G.R., BRASILEIRO, C.A., SCHIESARI, SCHIESARI, L., ALEXANDRINO, J. \& HADDAD, C.F.B. 2011. Anfíbios do Estado de São Paulo, Brasil: conhecimento atual e perspectivas. Biota Neotropica 11(1a): 1-19. http:// www.biotaneotropica.org.br/v11n1a/pt/abstract?inventory+bn004110 1a2011 (last access on 11/06/2017).

SÁ, R.O. \& LANGONE, J.A. 2002. The Tadpole of Proceratophrys avelinoi (Anura: Leptodactylidae). Journal of Herpetology 36(3): 490-494.

SÁ, R.O., LANGONE, J.A. \& SEGALLA, M.V. 2007. The tadpole of Leptodactylus notoaktites Heyer, 1978 (Anura, Leptodactylidae). South American Journal of Herpetology 2(1): 69-75.

SÁ, R.O., GRANT, T., CAMARGO, A., HEYER, W.R., PONSSA, M.L., \& STANLEY, E. 2014. Systematics of the neotropical genus Leptodactylus Fitzinger, 1826 (Anura: Leptodactylidae): phylogeny, the relevance of non-molecular evidence, and species accounts. South American Journal of Herpetology 9(s1): S1-S100.

SANTOS-PEREIRA, M., CANDATEN, A., MILANI, D., OLIVEIRA, F.B., GARDELIN, J. \& ROCHA, C.F.D. 2011. Seasonal variation in the leaflitter frog community (Amphibia: Anura) from an Atlantic Forest area in the Salto Morato Natural Reserve, southern Brazil. Zoologia 28(6): 755-761.

SANTOS-PEREIRA, M., ALMEIDA-SANTOS, M., OLIVEIRA, F.B., SILVA, A.L. \& ROCHA, C.F.D. 2015. Living in a same microhabitat should means eating the same food? Diet and trophic niche of sympatric leaf-litter frogs Ischnocnema henselii and Adenomera marmorata in a forest of Southern Brazil. Brazilian Jounal of Biology 75(1): 13-18.

SANTOS-PEREIRA, M. \& ROCHA, C.F.D. 2015. Invasive bullfrog Lithobates catesbeianus (Anura: Ranidae) in the Paraná state, Southern Brazil: a summary of the species spread. Revista Brasileira de Zoociências 16: 141-147.

SANTOS-PEREIRA, M. 2016. Comunidade de anfíbios anuros na Mata Atlântica da Reserva Natural Salto Morato, Paraná, sul do Brasil: diversidade, distribuição altitudinal e conservação. Tese de doutorado, Universidade do Estado do Rio de Janeiro, Rio de Janeiro.

SANTOS-PEREIRA, M., MILANI, D., BARATA-BITTENCOURT, L.F., IAPP, T.M. \& ROCHA C.F.D. 2016. Anuran species of the Salto Morato Nature Reserve in Paraná, southern Brazil: review of the species list. Check List 12(3): 1-11. doi: http://dx.doi.org/10.15560/12.3.1907 (last access on $11 / 06 / 2017)$. 
SEGALLA, M.V. \& LANGONE, J.A. 2004. Anfíbios. In Livro Vermelho da Fauna Ameaçada no Estado do Paraná. (S.B. Mikich, R.S. Bernils eds). Instituto Ambiental do Paraná, Curitiba, p.537-577.

SEGALLA, M.V., CARAMASCHI, U., CRUZ, C.A.G., GRANT, T., HADDAD, C.F.B., GARCIA, P.C.A., BERNECK, B.V.M. \& LANGONE, J.A. 2016. Brazilian amphibians: List of species. Herpetologia Brasileira 5: 34-46. http://www.sbherpetologia.org.br/images/LISTAS/Lista_Anfibios2016.pdf (last access on 11/06/2017).

SpeciesLink. 2017. http:// http://www.splink.org.brw (last access on 06/11/2017).

STEINBACH-PADILHA, G.C.A. 2008. New species of Melanophryniscus (Anura, Bufonidae) from the Campos Gerais region of Southern Brazil. Phyllomedusa 7(2): 99-108.

TOLEDO, L.F., GARCIA, P.C.A., LINGNAU, R. \& HADDAD, C.F.B. 2007. A new species of Sphaenorhynchus (Anura; Hylidae) from Brazil. Zootaxa 1658: $57-68$.

TOLEDO, L.F. \& BATISTA, R.F. 2012. Integrative study of Brazilian anurans: Geographic distribution, size, environment, taxonomy, and conservation. Biotropica 44(6): 785-792.
TOLEDO, L.F., GAREY, M.V., COSTA, T.R.N., MORAES, R.L., HARTMANN, M.T. \& HADDAD, C.F.B. 2012. Alternative reproductive modes of Atlantic forest frogs. Journal of Ethology 30: 331-336.

THOMÉ, M.T.C., ZAMUDIO, K.R., HADDAD, C.F.B. \& ALEXANDRINO, J. 2012. Delimiting genetic units in Neotropical toads under incomplete lineage sorting and hybridization. BMC Evolutionary Biology 12(242): 1-13.

TREIN, F.L., LIMA, L.P., ULANDOWSKI, L.K.A. \& MORATO, S.A.A. 2014. Pleurodema bibroni Tschudi, 1838 (Anura: Leiuperidae): Distribuition extension and first record for the state of Paraná, Brazil. Check List 10(2): 417-418.

VIEIRA, C.A., ALMEIDA, C.H.L.N., LAMBERTINI, C., LEITE, D.S. \& TOLEDO, L.F. 2012. First record of Batrachochytrium dendrobatidis in Paraná, Brazil. Herpetological Review 43(1): 93-94.

WINKELMANN, L. \& NOLETO, R.F. 2015. Caracterização cariotípica de Phyllomedusa tetraploidea (Anura, Hylidae), proveniente da Mata Atlântica Paranaense. Luminária 17(1): 146-157.
Received: $19 / 01 / 2017$

Revised: 04/04/2018

Accepted: 17/04/2018

Published online: 21/05/2018 